\title{
WestVirginiaUniversity
}

THE RESEARCH REPOSITORY @ WVU

Graduate Theses, Dissertations, and Problem Reports

2012

\section{Modeling and Analysis of GaN/InGaN Light Emitting Diodes}

\author{
Srinitya Musunuru \\ West Virginia University
}

Follow this and additional works at: https://researchrepository.wvu.edu/etd

\section{Recommended Citation}

Musunuru, Srinitya, "Modeling and Analysis of GaN/InGaN Light Emitting Diodes" (2012). Graduate Theses, Dissertations, and Problem Reports. 4900.

https://researchrepository.wvu.edu/etd/4900

This Thesis is protected by copyright and/or related rights. It has been brought to you by the The Research Repository @ WVU with permission from the rights-holder(s). You are free to use this Thesis in any way that is permitted by the copyright and related rights legislation that applies to your use. For other uses you must obtain permission from the rights-holder(s) directly, unless additional rights are indicated by a Creative Commons license in the record and/ or on the work itself. This Thesis has been accepted for inclusion in WVU Graduate Theses, Dissertations, and Problem Reports collection by an authorized administrator of The Research Repository @ WVU. For more information, please contact researchrepository@mail.wvu.edu. 


\title{
Modeling and Analysis of GaN/InGaN Light Emitting Diodes
}

\author{
by \\ Srinitya Musunuru \\ Thesis submitted to the \\ Benjamin M. Statler College of Engineering and Mineral Resources \\ at West Virginia University \\ in partial fulfillment of the requirements \\ for the degree of \\ Master of Science \\ in \\ Electrical Engineering \\ Dimitris Korakakis, Ph.D. \\ Jeremy M. Dawson, Ph.D. \\ Lawrence A. Hornak, Ph.D., Chair
}

Lane Department of Computer Science and Electrical Engineering

Morgantown, West Virginia

2012

Keywords: Tunneling Contacts,Sentaurus, GaN/InGaN, ZnO

Copyright 2012 Srinitya Musunuru 


\author{
Abstract \\ Modeling and Analysis of GaN/InGaN Light Emitting Diodes \\ by \\ Srinitya Musunuru \\ Master of Science in Electrical Engineering \\ West Virginia University \\ Lawrence A. Hornak, Ph.D., Chair
}

GaN based optoelectronic devices have had significant impact in solid state lighting. Developing efficient light emitting diodes has been of great research interest in recent years. Electrical modeling of light emitting diodes is now gaining its importance with the development of TCAD tools to have a better understanding of the device structure and to have cost reduction associated with the material and labor.

In this thesis a TCAD model for our device has been developed with the finite element analysis TCAD tool sentaurus from synopsys. The developed model has been validated to the experimental results. The electrical characteristics of the device have been analyzed with the use of band diagrams, current distribution, radiative recombination rate and IV plots.

Different layers of the device have been studied and analyzed and certain design changes to achieve an enhancement in the efficiencies are proposed. The problem of current crowding in LEDs has been widely reported in the literature. In this study passive modeling of the LED structure with PSPICE has been carried out to understand the impact of the conductivities of different layers on the problem of current crowding. With the feedback from the PSPICE model an analytical relation has been determined between the p-GaN layer and n-GaN layer to have uniform current spreading. However, establishing the analytical relation between these layers is experimentally challenging. An alternate design change utilizing transparent conducting AZO contact to p-GaN has been designed and analyzed. 


\section{Acknowledgements}

It's my pleasure to thank all the people involved in my thesis.

I owe my deepest gratitude to my advisor Dr. Lawrence A Hornak, for his continuous support, encouragement and guidance through my master's. This thesis is possible only due to his masterly guidance. His extensive knowledge and vision has been the source of inspiration for my work. I strongly believe that my potential as a professional engineer has increased multifold during my association with Dr.Hornak.

I am very much grateful to my committee members Dr. Dimitris Korakakis and Dr. Jeremy Dawson for their encouragement, technical discussions and feedback.

Immense thanks to my labmates Dr.Sridhar Kuchibhatla, Dr. Lee Rodak, Dr. Kyoungnae Lee, Joshua Justice, Rohit Goswami, Vamsi Kumbham, Anand Kadiyala, Vishal Narang, Bashar Hamza, Benjamin Bearce and Kenneth Hite for their help and knowledge exchange in this project.

I owe my sincere thanks and dedicate this thesis to my parents Mr. Vishnu Vardhana Rao and Mrs. Gangabhavani, for all their support and encouragement through my childhood.

Special thanks to my friends Ravindra Gadde, Neeharika Chennupati, Raghu Yalamanchili, Maurya Srungarapu and Sriram Thotakura for all their support and encouragement during my stay away from home.

Last but not the least I thank lord almighty for blessing me with such wonderful people in my life. 


\section{Contents}

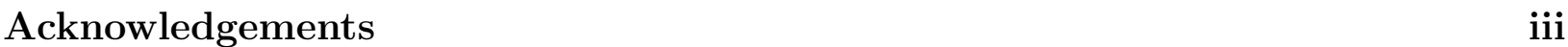

List of Figures $\quad$ vi

List of Tables $\quad$ viii

$\begin{array}{ll}\text { Notation } & \text { ix }\end{array}$

1 Introduction $\quad 1$

1.1 Introduction . . . . . . . . . . . . . . . . . . . . 1

1.1.1 Scope of this Thesis . . . . . . . . . . . . . 8

2 Theory $\quad 10$

2.1 Semiconductor Physics . . . . . . . . . . . . . . . . . . . 10

2.2 Conduction and emission mechanisms in semiconductors . . . . . . . . . . 12

2.3 Principles of p-n junction diode . . . . . . . . . . . . . . . . . . 15

2.3.1 Bias conditions for p-n junction . . . . . . . . . . 16

2.3.2 Non-Idealities in a p-n junction . . . . . . . . . . . . . . . . . . . 19

2.4 Metal-Semiconductor Junctions . . . . . . . . . . . . . . . . . . . . . . . . 19

2.4.1 Schottky Contacts . . . . . . . . . . . . . . . 20

2.4 Tunneling contacts . . . . . . . . . . . . . . . 22

2.4 Ohmic Contacts . . . . . . . . . . . . . . . . . 22

2.4.4 Surface effects on metal-semiconductor contacts . . . . . . . . . . 23

2.5 Quantum Well . . . . . . . . . . . . . . . . . . . . . . . . . . . . . . . . . . . 23

2.6 Light Emitting Diode . . . . . . . . . . . . . . . . . . . . . . . . . . 24

2.6.1 Choice of Materials . . . . . . . . . . . . . . . . . . 25

2.6.2 Recombination Mechanisms in a LED . . . . . . . . . . . . . . 26

2.6.3 Efficiency ......................... 28

3 Physics and Simulation of Light Emitting Diodes $\quad 29$

3.1 Carrier Transport . . . . . . . . . . . . . . . . . . . 32

3.1.1 Carrier transport models:Drift and Diffusion . . . . . . . . . . . 32

3.1.2 Boundary conditions for solving continuity equations . . . . . . . . 33

3.2 Generation-Recombination Mechanisms in LEDs . . . . . . . . . . . . . . . 35

3.2.1 Radiative Recombination . . . . . . . . . . . . . . . . . . 35 
3.2.2 Auger Recombination . . . . . . . . . . . . . . . . . . 35

3.2 .3 Shockley-Hall Recombination . . . . . . . . . . . . . . . 36

3.3 Ray Tracing . . . . . . . . . . . . . . . . . . . . . 36

4 Modeling the LED $\quad 38$

4.1 Structure and device parameters used for this study . . . . . . . . . . . . . 38

4.1.1 LED structure used for this study . . . . . . . . . . . . . . . . . 38

4.1.2 Material parameters used for this study . . . . . . . . . . . . . 39

4.2 Preliminary Modeling Results . . . . . . . . . . . . . . . . . . . . . . . . . . . . . . . . . . . . 40

4.2 .1 Band Structure . . . . . . . . . . . . . . . . . 40

4.3 Validation of the developed model . . . . . . . . . . . . . . . . . . 43

4.4 Analysis of effectiveness of AlGaN layer for carrier confinement . . . . . . . . 45

4.4.1 Composition Variation . . . . . . . . . . . . . . . . . . . . 45

4.5 Influence of thickness . . . . . . . . . . . . . . . . . . . . 47

5 Design analysis $\quad 49$

5.1 Drawbacks of the previous structure . . . . . . . . . . . . . . 49

5.1 .1 Analysis of the structure . . . . . . . . . . . . . . . 50

5.2 Design changes to $\mathrm{p}-\mathrm{GaN} \ldots \ldots \ldots \ldots \ldots \ldots$

6 Potential of $\mathrm{AZO}$ as transparent conducting contact to p-GaN 57

6.1 Design of AZO as transparent contact to $\mathrm{p}-\mathrm{GaN} \ldots \ldots \ldots$. . . . . . 57

6.2 Analysis of AZO contact o p-GaN . . . . . . . . . . . . . . . . 59

7 Conclusions and Future work $\quad 63$

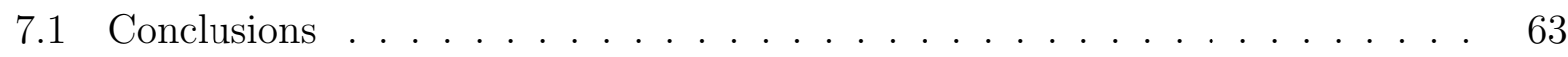

7.2 Future Work . . . . . . . . . . . . . . . . . . . 64

$\begin{array}{ll}\text { References } & 66\end{array}$ 


\section{List of Figures}

1.1 Band gap engineering of the III-Nitride materials to achieve the desired wavelength of devices $[1] . \ldots \ldots \ldots \ldots$. . . . . . . . . . . . . . . 4

1.2 Single Quantum Well heterostructure LED [2]. . . . . . . . . . . . . . . . 5

1.3 Proposed Flip chip LED by Wierer et'al [3] . . . . . . . . . . . . . . . 6

1.4 The top view of the Photonic crystal embedded on the LED as illustrated by Barton et'al $[4] \ldots \ldots \ldots \ldots \ldots$. . . . . . . . . . . . . . . . . . . 6

1.515 period $\mathrm{GaN} / \mathrm{Al}_{0} .27 \mathrm{Ga}_{0} .73 \mathrm{~N}$ DBR on Sapphire substrate for InGaN/GaN multi quantum well LED as illustrated by Nakada et'al [5]. . . . . . . . . 7

1.6 ITO as transparent conducting layer to InGaN/GaN LED Lim et'al [6]. . . . 8

2.1 Band diagrams of 1)Insulators 2)Semiconductors 3)Metals. . . . . . . . . . . 11

2.2 E-K diagram illustrating direct and indirect band gap semiconductors[7] . . 12

2.3 Illustration of band diagram, available states, probability of occupancy of a state, distribution of carriers [8]. . . . . . . . . . . . . . 14

2.4 Stimulated and Spontaneous Emission Mechanisms [7]. . . . . . . . . . . 15

2.5 P-N junction under bias conditions. . . . . . . . . . . . . . . . 17

2.6 Band diagram of $\mathrm{p}-\mathrm{n}$ junction diode under forward bias conditions. . . . . . 17

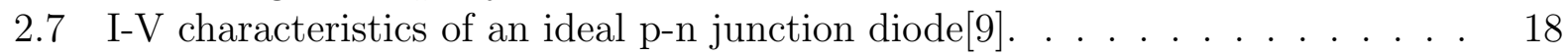

2.8 I-V characteristics of a $\mathrm{p}-\mathrm{n}$ junction diode considering the impact of series and parallel resistances[9] . . . . . . . . . . . . . . . . . . . 20

2.9 Band diagrams of Schottky contacts on $\mathrm{p}$ and n-type semiconductors[10]. . . 21

2.10 Band diagrams of Ohmic contacts on $\mathrm{p}$ and n-type semiconductors [10]. . . . 22

2.11 Basic light emitting diode. . . . . . . . . . . . . . . . 24

2.12 Radiative recombination with the emission of a photon and non-radiative recombination creating vibrations in the lattice $[9] . \ldots \ldots . . . . . . .27$

4.1 LED structure employed for modeling. . . . . . . . . . . . . . . . . 38

4.2 Band diagram of the LED device plotted along the vertical direction of the LED. . . . . . . . . . . . . . . . . . . . . 40

4.3 Structure and band diagram of the LED device without the p-AlGaN layer plotted along the lateral direction of LED. . . . . . . . . . . . . . . . . 41

4.4 Electron and hole distribution along the LED from p-GaN to n-GaN. . . . . 42

4.5 Radiative recombination along the LED from p-GaN to n-GaN. . . . . . . . 42

4.6 Mask used to fabricate the LEDs. . . . . . . . . . . . . . . . . . . . 43 
4.7 Simulated and experimental IV plots for different LEDs (a) varying the pcontact size with the spacing between p-mesa and n-contact being 25 microns (b) varying the spacing between $\mathrm{p}$-mesa and n-contact with the p-contact size 100microns. . . . . . . . . . . . . . . . . .

4.8 Resistance plots comparison for different devices with a p-contact size of 100microns, varying the space between p-mesa and n-contacts. . . . . . . . . 44

4.9 Band diagram for $\mathrm{x}=0.2$ in $\mathrm{Al}_{x} \mathrm{Ga}_{1-x} \mathrm{~N} \ldots \ldots \ldots \ldots \ldots \ldots$. . . . . . . . . . 45

4.10 Band diagram for $\mathrm{x}=0.2 \mathrm{x}=0.1$ and $\mathrm{x}=0.05$ in $\mathrm{Al}_{x} \mathrm{Ga}_{1-x} \mathrm{~N}$. . . . . . . . 46

4.11 Electron distribution, hole distribution and radiative recombination rates in the LED with varying $\mathrm{Al}$ composition. . . . . . . . . . . . . . . . . . . 46

4.12 Band diagram for the LED structure varying the thickness of the EBL to be 10nm, 20nm and 40nm. . . . . . . . . . . . . . . .

4.13 Electron, hole and radiative recombination rate distributions in the quantum wells. . . . . . . . . . . . . . . . . . . . .

5.1 Current density distribution plot (a) along the lateral direction of the LED in the first quantum well for the structure shown (b) . . . . . . . . . . . . . . 49

5.2 Resistance model representation of the LED . . . . . . . . . . . . . . . . 51

5.3 Simplified resistance model representation of the LED. . . . . . . . . . . . 52

5.4 PSPICE modeling of the resistance model for the condition $\mathrm{R}_{p}>\mathrm{R}_{n}, \mathrm{R}_{n}>$ $\mathrm{R}_{p}, \mathrm{R}_{n} \approx \mathrm{R}_{p}, \mathrm{R}_{n}=\mathrm{R}_{p} \ldots \ldots \ldots \ldots$

5.5 Illustration of increase in the efficiency of $140 \mathrm{~nm}$ thick $\mathrm{p}-\mathrm{GaN}$ over $70 \mathrm{~nm}$ thick p-GaN . . . . . . . . . . . . . . . . . .

6.1 Calculated AZO/p-GaN junction depletion widths for varying hole concentration in the $\mathrm{p}-\mathrm{GaN}$ layer. . . . . . . . . . . . . . . . 58

6.2 Band diagram indicating $\mathrm{Ni} / \mathrm{AZO}$ junction. . . . . . . . . . . . . . . . 59

6.3 IV plots indicating Ni/AZO junction with varying Ni thickness. . . . . . . . 60

6.4 Current distribution in the LED mesa with AZO initial parameters. . . . . . 60

6.5 Current distribution in the LED mesa with varying thickness of AZO . . . . 62 


\section{List of Tables}

4.1 Experimentally extracted material parameters . . . . . . . . . . . . . . . 39

4.2 Material parameters from literature . . . . . . . . . . . . . . . . . . 39 


\section{Notation}

We use the following notation and symbols throughout this thesis.

$L E D \quad$ : Light emitting diode

$M Q W \quad$ : Multi quantum well

( $\rho) \quad:$ Resistivity

$(\nu) \quad:$ Mobility

$(\chi) \quad:$ Electron Affinity

DUT ; Device under test

$(\epsilon) \quad$ : Epsilon

$E B L \quad$ : Electron blocking layer

$A Z O$ : Aluminium alloyed Zinc Oxide 


\section{Chapter 1}

\section{Introduction}

\subsection{Introduction}

Since, the invention of the incandescent bulb by Thomas Edison in 1979, there has been great thirst to create less expensive, more reliable and brighter light sources. Massive industries have been created to produce filament and fluorescent lamps for interior decoration, sodium discharge lamps for street lighting etc. Given, the drawbacks associated with the efficiency and life time of these sources, solid state lighting, based on semiconductor light emitting diodes had made a revolutionary change with their applications in a wide variety of fields. WARP 75 consisting of a number of LEDs is device developed by NASA that is being used as source of light for plants grown on space station and the biologists have found that cells exposed to near infrared light have grown 150 to 200 times faster than those not simulated by any such light [11]. This device is now being used in medical applications and is believed to improve the bone marrow transplant patient's quality of life[12]. LEDs are now being used in automobile applications and the introduction of LED headlights by Audi is expected to revolutionize the automotive industry. The key for all these applications is developing efficient LEDs.

Light emitting diodes are specially designed semiconductor $p-n$ junctions that act as transducers converting electrical energy to light energy, with the process known as electroluminescence. The color of light emitted mainly depends on the band gap of the designed 
p-n junction, given the relation:

$$
\text { Energy of the emitted photon, } E=\frac{h c}{\Lambda}
$$

The history of LED technology dates back to the 1920's with the introduction of SiC based LED's. However, the efficiency of these devices was less than $0.005 \%$ thus, raising the need for alternating materials [9]. The growth of III-V light emitting diodes started in 1954. The III-V compounds serve as promising materials for the growth of LED's because of their wide band gap property. GaAs based infrared light emitting diodes were reported in 1962 by groups from RCA, GE, IBM and MIT. The maximum efficiencies achievable with these materials was around 0.2-0.35\% [9]. Visible LEDs were realized by alloying GaAs and $\mathrm{GaP}[13]$. The limiting efficiency of these alloy devices was $0.2 \%$ for GaAs and a droop to a value less than $0.005 \%$ with increase in phosphorous to $44 \%$ [14].

AlInGaP has gained in importance for light emitting diode applications. The performance of AlInGaP based LEDs is characterized by a green emission wavelength peaking around 570nm with an efficiency of around 1\%[15]. Efforts to move the emission peak towards greenish light resulted in the reduction in the external quantum efficiency as the band structure of AlInGaP approaches indirect transition band structure.

In 1960 the director of the Radio Corporation of America, James Tietjen and his group started exploring GaN to realize visible LEDs. In 1969 the successful growth of single crystal GaN on sapphire has been reported by Maruska and Tietjen of RCA laboratories [16]. The GaN powder was prepared by reacting ammonia with liquid gallium metal, as indicated in the following reaction :

$$
2 \mathrm{Ga}+2 \mathrm{NH}_{3} \rightarrow 2 \mathrm{GaN}+3 \mathrm{H}_{2}
$$

GaN samples obtained were found to be unintentionally doped n-type. Achieving p-type GaN then became an area of intense research interest to obtain a GaN based p-n junction. Zinc was first explored to be used as a p-type dopant to GaN, however the GaN sample turned insulating with zinc dopants. The first GaN LED was still fabricated by Pankove et al that consisted of an undoped n-type region, an insulating Zn doped layer and an Indium 
surface contact [17]. The realized LED emitted red, blue, green or yellow light depending on the Zinc concentration.

Later in 1972, Maruska et al proposed the use of magnesium dopants over zinc dopants [18]. GaN LEDs having emission wavelength of around 430nm were obtained with magnesium dopants. Since then, magnesium dopants have since become the basis for all commercially available LEDs. However, obtaining good conducting GaN films with magnesium dopants was still a challenge, and the research virtually ceased because of the difficulties invloved. Later, in 1989 Amano et al eventually was able to obtain good p-type GaN, and reported that low energy beam radiation could help to obtain a two order increase in the photo luminescence [19]. Van Vechten et al attributed this increase in p-type conducting nature to the formation of an $\mathrm{Mg}: \mathrm{H}$ complex that passivates the acceptor and prohibits p-type conduction. When irradiated with a low energy electron beam the complex and enables Magnesium to settle as a shallow acceptor at approximately $0.16 \mathrm{eV}$ above the valence band $[20]$.

GaN having a band gap of $3.4 \mathrm{eV}$, is alloyed with other III-Nitride materials to form a large range of direct band gap materials, making it a promising material for applications in various optoelectronic devices [15]. GaN based LED's employ band gap engineering techniques, wherein the elemental composition of semiconductor alloys is varied in a controlled way to achieve a desired band gap that can emit in the desired range of wavelengths.

In 1995, Shuji Nakamura from Nichia Industries Corporation, Japan demonstrated the development of first blue and green InGaN based double heterostructure LEDs with efficiencies of around $10 \%$ by band gap engineering of InN and GaN percent composition [21]. In this study, InGaN quantum wells used were obtained by alloying InN with GaN, with which an emission range of $1.0 \mathrm{eV}$ to $3.4 \mathrm{eV}$ could be obtained.

$$
x(G a N)+1-x(\operatorname{InN}) \rightarrow \operatorname{In}_{1-x} G a_{x} N
$$

Since then, the ternary III-Ntiride semiconductor compound InGaN, has become the candidate for use as the active layer in Quantum wells. Thus GaN based optical device struc- 


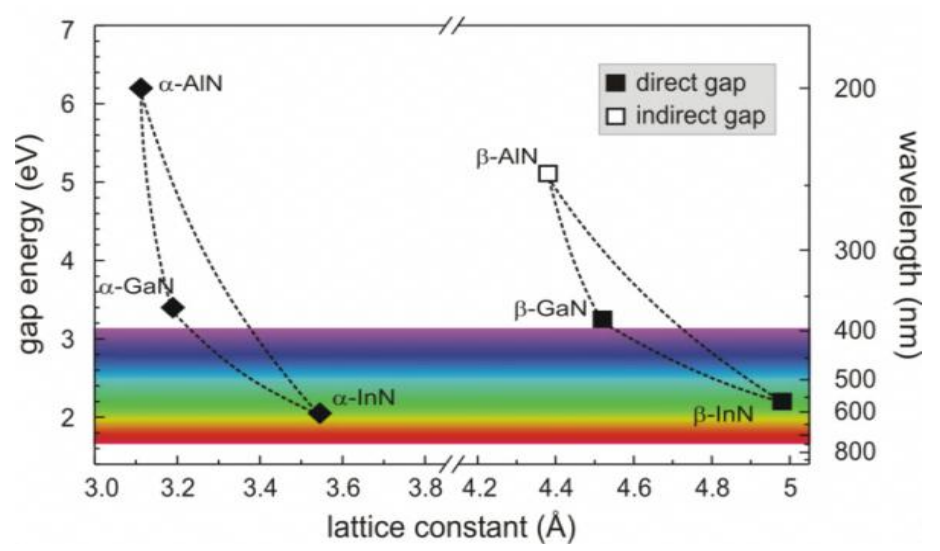

Figure 1.1: Band gap engineering of the III-Nitride materials to achieve the desired wavelength of devices [1].

tures take advantage of GaN/AlGaN, GaN/InGaN Quantum Wells. The InGaN films were grown by two-flow MOCVD method. Trimethylgallium (TMG), trimethylaluminum(TMAl), trimethylindium (TMI), monosilane (SiH4), bis-cyclopentadienyl magnesium (CpzMgj, diethylzinc (DEZ), and ammonia (NH,) were used as $\mathrm{Ga}, \mathrm{Al}, \mathrm{In}, \mathrm{Si}, \mathrm{Mg}, \mathrm{Zn}$, and $\mathrm{N}$ sources, respectively [2].

In the first double heterostructure LED as shown in fig 1.2, all the layers as reported by Nakamura et'al were grown by MOCVD. Ni/Au layer was directly evaporated on to p-GaN to obtain the p-contact while, the $\mathrm{Ti} / \mathrm{Al}$ contact has been evaporated to $\mathrm{n}$-GaN upon etching the p-GaN and the quantum wells [2].). An external efficiency of $2.7 \%$ has been obtained using this LED.

Several investigations have then been made on use of InGaN/GaN Multi Quantum Well structures as opposed to bulk InGaN, and an enhanced optical efficiency of nearly 2 orders of magnitude greater was obtained [22]. The external quantum efficiencies of blue and green MQW LEDs was increased to $10 \%$ and $7 \%$ respectively.

The major challenge in the research and development of designing the LEDs includes developing structures to minimize the input electrical power, and generate more light output 


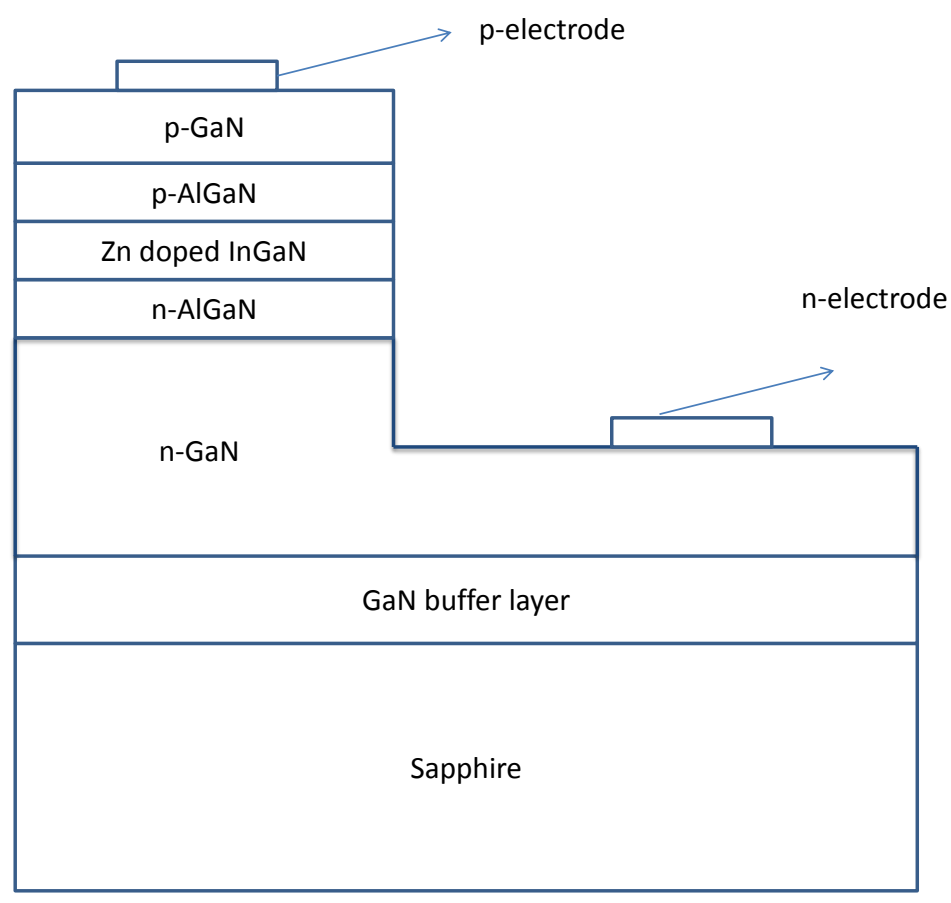

Figure 1.2: Single Quantum Well heterostructure LED [2].

with minimal heat dissipation i.e increase the internal efficiency or the electroluminescence and the extraction efficiencies [9].

The improvement of internal quantum efficiency of the device greatly depends on the design of the LED and specifically on the layer characteristics and the quality of the material growth. The dependence of the internal quantum efficiency on the layer characteristics of the LED will be covered as a part of this thesis.

It has been observed that, an LED with a high internal efficiency still had a lower external efficiency, given the problem of total internal reflection in LEDs [9]. There have been various design changes proposed to enhance the external quantum efficiency some of which are now discussed.

Wierer et'al reported flip chip LEDs with 1.6 times more external extraction efficiency than a conventional top emitting LED. The major disadvantage of the conventional LEDs is the absorption of photons by the metal contacts, bond pads and wire bonds to the package. In the proposed flip-chip design, the light emitting chip is inverted compared to the conventional LEDs, thus avoiding absorption from the contact pads, as the extraction is through the transparent Sapphire substrate [3]. 


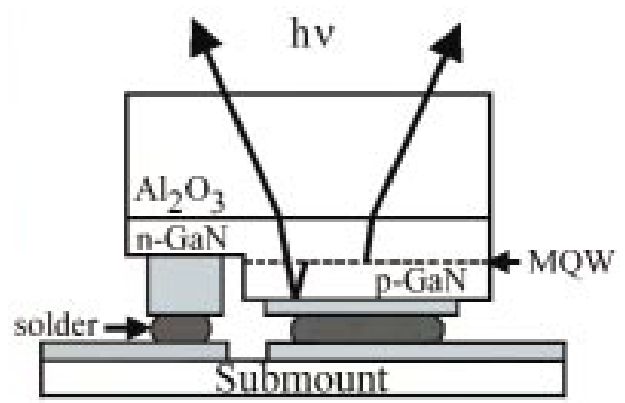

Figure 1.3: Proposed Flip chip LED by Wierer et'al [3]

Another major advancement in improving the extraction efficiency of LEDs has been introduced by Boroditsky et'al, with the use of a two dimensional photonic crystal on LEDs. A thin slab of photonic crystal can be used to tailor the emission direction so as to have increased emission in the useful directions and reduce the need for noisy loss reflectors. Efficiencies of 1.5-2 times higher than conventional LEDs have been reported [23].

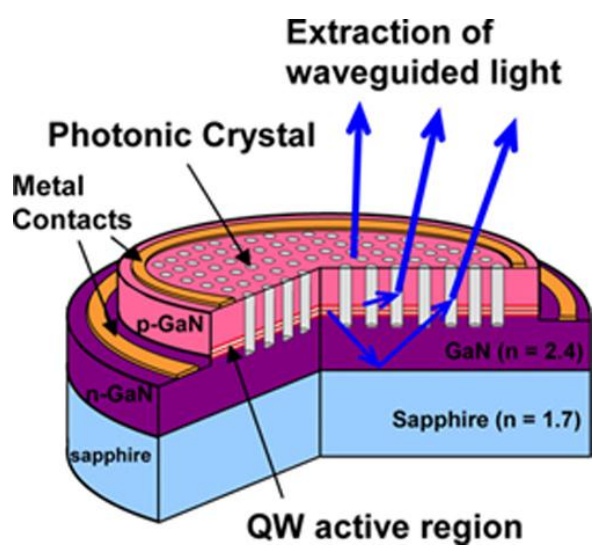

Figure 1.4: The top view of the Photonic crystal embedded on the LED as illustrated by Barton et'al [4]

Nakada et'al in 2000 reported that, a DBR introduced before the fabrication of LED could improve the extraction efficiency of the device, given the fact that the transparency of Sapphire substrate could cause loss of light. A 30\% increase in the external quantum efficiency has been observed with the use of 15 period GaN/AlGaN DBR structures [5].

Also, to have better performance characteristics it is quite important to get a very good contact system to the materials, to minimize the voltage drop at the metal-semiconductor 


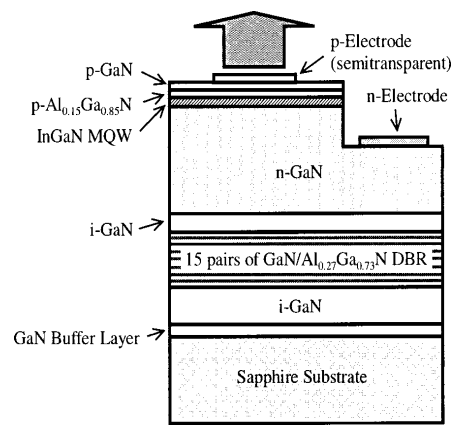

Figure 1.5: 15 period GaN/ $\mathrm{Al}_{0} .27 \mathrm{Ga}_{0} .73 \mathrm{~N}$ DBR on Sapphire substrate for InGaN/GaN multi quantum well LED as illustrated by Nakada et'al [5].

junction. Ohmic contact system to $\mathrm{n}-\mathrm{GaN}$ can be easily formed by using a metal with work function less than that of GaN. Ti-Al based contact schemes have been widely used for n-GaN [20].

Achieving less resistive ohmic contacts to the p-GaN has been a great challenge for the fabrication of LEDs, given the large work function of p-GaN due to its high electron affinity and large band gap. The formation of ohmic contacts thus needs either a low schottky barrier or a heavily doped p-GaN through which the carriers could tunnel. Knowing the difficulties in incorporating magnesium dopants in $\mathrm{p}-\mathrm{GaN}$ it is easier to achieve a low schottky barrier by proper annealing of the metal contacts. Annealed Ni/Au contacts have been used since several years to form a good contact system to p-GaN. Koide (et al) described the ohmic characteristics of the annealed $\mathrm{Ni} / \mathrm{Au}$ contacts to the formation of semiconductor layer with high carrier concentration formed due to the removal of hydrogen bonded with Magnesium or Nitrogen in GaN [24]. Several studies later reported the key for formation of ohmic contacts to $\mathrm{p}-\mathrm{GaN}$ is some interfacial reactions resulting in interfacial compounds of Gallium with Nickel and Gold.These metal contacts were however, only partially transparent, and most of the light generated cannot be extracted from the top of devices, despite of the fact that GaN based LEDs are optically transparent. Kim et'al thus proposed that, the use of transparent conducting ITO as ohmic contacts to p-GaN, would increase the light emitting area, thereby 
increasing the intensity of the light extracted and hence the external quantum efficiency [25]. Since, then several transparent conducting oxides such as Cadmium Tin Oxide, Zinc Oxide have been studied and reported [6].

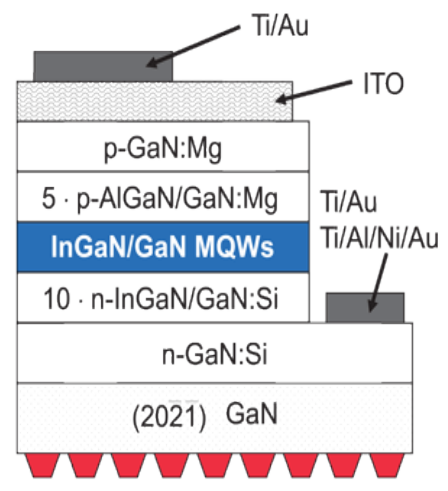

Figure 1.6: ITO as transparent conducting layer to InGaN/GaN LED Lim et'al [6].

\subsubsection{Scope of this Thesis}

Designing light emitting diodes to have better efficiency is a great challenge to researchers in this area. This thesis deals with the study of the impact of various layers in the structure and discusses the impact of various design changes to the carrier confinement in LED. Apart from the experimental methods, computational methods for design and evaluating of the light emitting diodes play a vital role in research and development. Once tuned to experimental results from a particular device these tools, enable prediction of the impact of device design changes on performance resulting in cost savings. The various TCAD tools APSYS from Crosslight, Sentaurus from Synopsys, Silvaco etc. available in the market have made computational modeling of the semiconductor devices like LEDs, LASERs, FETs, MOSFETs easier.

In this thesis, the finite element analysis tool Sentaurus from Synopsys has been used to study the physics and the important design aspects of LEDs. The models used for the device modeling are the basic electromagnetic wave equations based on the poisson's 
equation, the continuity equations for the carrier transport, to solve the electrical problems and gain equations based on schrodinger's equation are used to couple the electrical and optical problems and some recombination models, that will studied in detail in the coming chapters.

In this study, MOCVD grown GaN and InGaN layers with, etched circular mesa structures is employed.The material physical parameters are first tuned to match the simulated results more closely with the experimental results. These results were tested and validated by comparing the IV's, band structure's, current density distributions and optical power measurements, and will be discussed and explained in detail to get a better understanding of the LED physics.

The impact of AlGaN electron blocking layer on the carrier confinement of electron-hole pairs to the multi quantum well has been studied in detail and the influence of design changes to this layer on the device characteristics has been studied and presented in this thesis.

The problem of current crowding with $\mathrm{Ni} / \mathrm{Au}$ contact on LEDs has been studied. An analytical model has been developed using SPICE tools to understand the dependence of current crowding on conductivities of each of the layers, and design changes to p-GaN have been proposed. Alternatively aluminium alloyed zinc oxide has been designed for use as a transparent conducting contact to p-GaN and techniques to achieve uniform current spreading with such a contact is discussed. 


\section{Chapter 2}

\section{Theory}

\subsection{Semiconductor Physics}

This chapter deals with the basic material properties particularly the semiconductors, the basics of semiconductor devices, p-n junction diodes and optoelectronic devices specifically the light emitting diodes. Materials are broadly classified into three different classes; conductors, insulators and semiconductors [26].

Conductors: A conductor is a material, in which the outer electrons of the atom are loosely bound and are free to move through the material. Such materials usually have a overlapped conduction and valence bands. Metals are the best examples of conductors.

Insulators: An insulator is a material in which the atoms hold the outer electrons tightly. Such materials have considerable a large energy gap between the conduction and the valence bands. Some examples of the insulators include glass, plastic, rubber etc.

Semiconductors: A semiconductor is a material whose conductivity lies between conductors and insulators. Semiconductors could be either elemental or compound semiconductors. Elemental semiconductors include silicon, germanium etc; while, GaN, GaAs etc; are the examples of compound semiconductors. The electronic properties of a semiconductor could be controlled by the addition of external impurities called dopants. Pure semiconductors are called intrinsic semiconductors, while semiconductors with external impurities are called extrinsic semiconductors. The extrinsic semiconductors are usually classified as p or n-type semiconductors, depending on if the kind of dopants is acceptors, carrier creating a fixed 


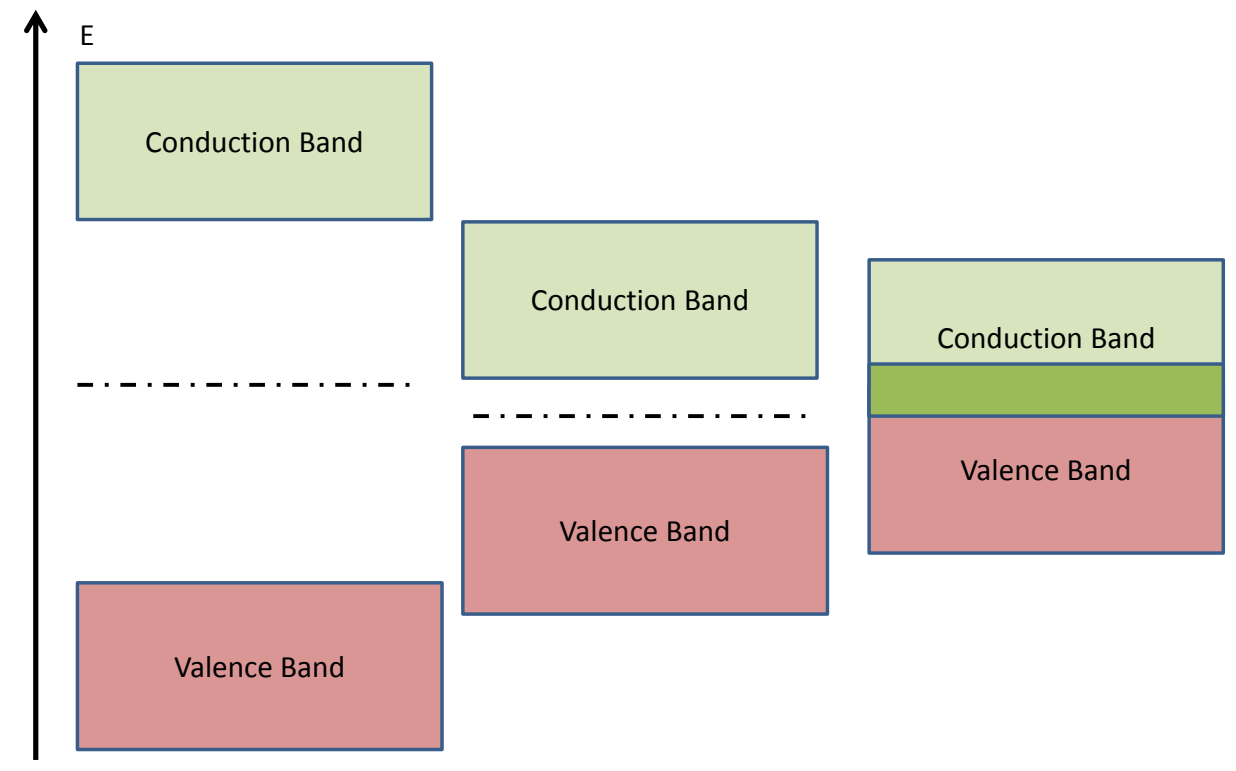

Figure 2.1: Band diagrams of 1)Insulators 2)Semiconductors 3)Metals.

negative charge at the dopant site and a mobile hole in the lattice, or donates an electron creating abundance of holes or a fixed positive charge and a mobile electrons respectively, respectively. Extrinsic semiconductors and their dopant control in quality and location forms the basis of many semiconductor device applications.

The E-K diagram or energy-momentum diagrams play a very important role in determining the electrical properties of a given material. The band gap energy, fermi level and the electron affinity are the characteristic of each material and are the basis of the band diagrams. The band gap energy is the minimum energy difference between the valence band and the conduction band and is nearly zero for conductors, reasonably high for insulators such that there are no free carriers at room temperature and intermediate for semiconductors. The fermi level denotes the highest occupied level by an electron in the valence band and the electron affinity dictates the amount of energy required to remove an electron from the conduction band of the solid into vacuum.

Semiconductor materials are further classified as direct and indirect band gap materials, depending on the position of the conduction band maxima and the valence band minima. 

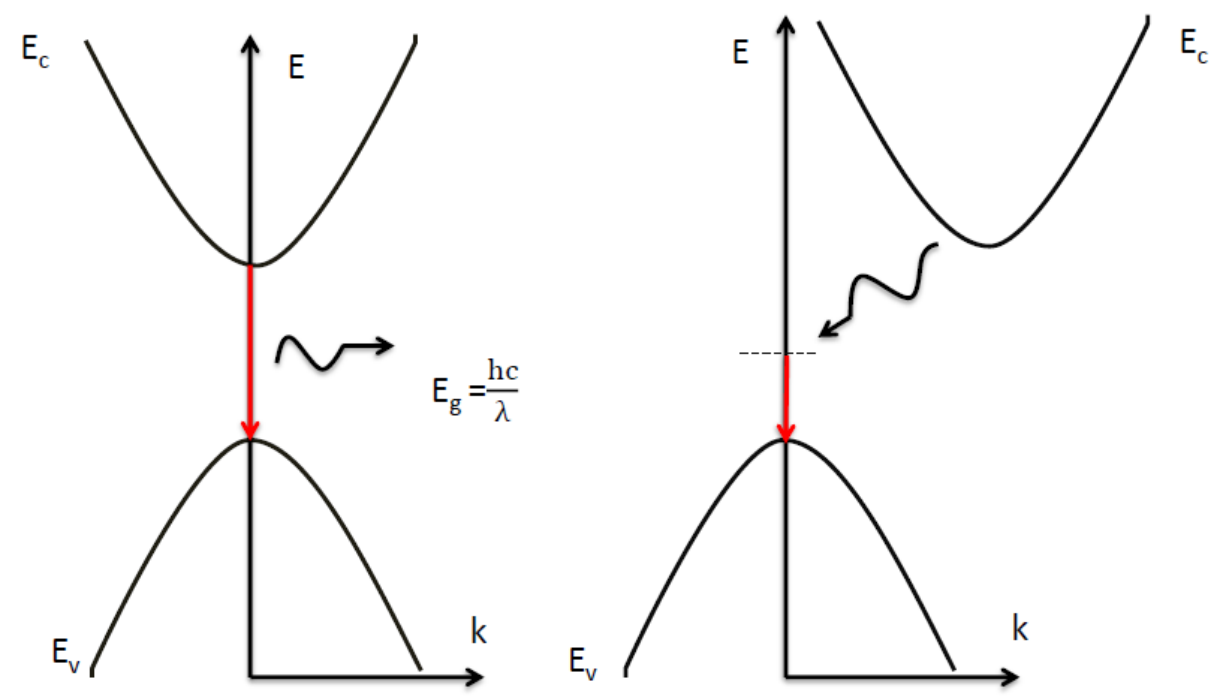

Figure 2.2: E-K diagram illustrating direct and indirect band gap semiconductors[7]

A material is said to be direct band gap material, if the conduction band minima and the valence band maxima of the semiconductor have the same k-vector and an indirect band gap semiconductor if there is a displacement in the k-vectors of the conduction band minima and the valence band maxima. In the case of a direct band gap semiconductor an electron in the conduction band minima can directly recombine with a hole in the valence band maxima without the need for an intermediate transition, while an indirect band gap semiconductor involves an intermediate transition to preserve the law of conservation of momentum. The EK diagrams examples of direct and indirect band gap materials are shown in the Figure2.2[7].

\subsection{Conduction and emission mechanisms in semicon- ductors}

In a semiconductor at $0 \mathrm{~K}$, the conduction band is empty and the valence band is completely filled with electrons, thus conducting no electricity. As temperature increases, electrons may overcome the forbidden energy or the band gap energy and reach the conduction 
band, leaving behind holes near the top of the valence band, with the process known as an intrinsic generation process. Under the influence of an external electric field, both the carriers tend to move, in opposite direction and start conducting.

The electrons and holes are distributed into the conduction band and valence band and, are governed by the Fermi-Dirac statistics assuming that no two two particles can occupy the same state. The Fermi-Dirac distribution function, indicates the probability that a state of energy E could be filled with an electron and is denoted by [26]

$$
f(E)=\frac{1}{1+\exp \left[\left(E-E_{f}\right) / k t\right]}
$$

If $g(E)$ denotes the number of states available for occupancy, $f(E)$ denoting the probability of occupying a state, the distribution of electrons in an energy interval $\mathrm{dE}$ is given by,

$$
\begin{aligned}
& d n(\mathbf{E})=\rho_{c}(\mathbf{E}) \cdot f_{n}(\mathbf{E}) \cdot d(\mathbf{E}) \\
& d p(\mathbf{E})=\rho_{v}(\mathbf{E}) \cdot f_{p}(\mathbf{E}) \cdot d(\mathbf{E})
\end{aligned}
$$

The basis of operation of any semiconductor device is the creation or annihilation of electron-hole pairs that involves, exciting an electron from valence band to conduction band. In principle any energetic particle, incident on the semiconductor which could impart energy equal to the band gap energy could results in excitation of electrons from the valence band to the conduction band causing, current to flow, making the system thermodynamically unstable, $\mathrm{np}=n_{i}^{2}$, deviating from the equilibrium state. To attain the stability, the excited electrons tends to fall back to a lower energy band say the valence band, releasing the excess energy, which usually is the band gap energy in the form of a photon or phonon the process being either radiative or non-radiative recombination. The characteristics of the photon are governed by the equation:

$$
\text { Energy of the emitted Photon, } \triangle E=h c / \Lambda
$$

where, $\triangle E$ is the energy difference between the two states.

This emission mechanism of an unstable system could be either spontaneous emission or stimulated emission. In stimulated emission, an incoming photon, stimulates the whole 


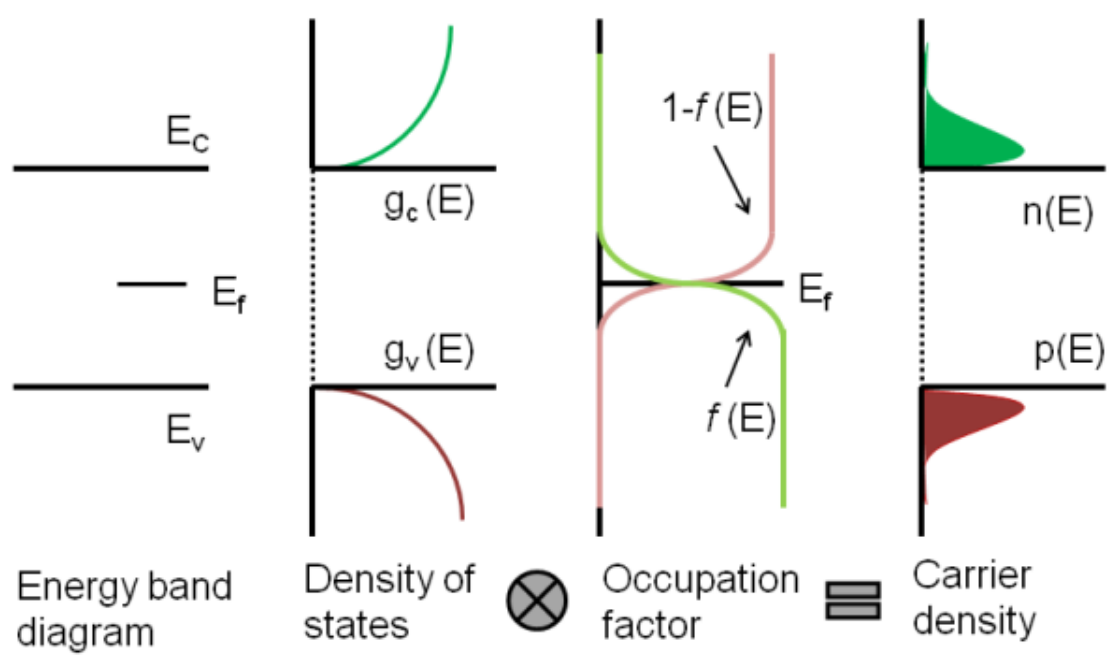

Figure 2.3: Illustration of band diagram, available states, probability of occupancy of a state, distribution of carriers [8].

emission, by inducing the electron at E2 to transit down to E1. On the other hand, in spontaneous emission, an electron at a higher energy state E2, falls back to a lower energy state E1, with the emission of excess energy as a photon. The difference between the two states is equal to the energy of the photon and determines the wavelength of the emitted photon. Also, in stimulated emission due to the coupling of the electric fields of the photon, and the electron, the emitted photon is in phase with the incoming photon, and has the same polarization and energy as the incoming photon. For stimulated emission, no electron in the E1 state, should absorb the photon. For this to happen, the number of electron in the excited state E2, should be greater than the number of electrons in E1 state, also known as population inversion, while there is no such requirement for spontaneous emission. Spontaneous emission forms the principle of operation of LEDs, while the stimulated emission forms the basis of Laser diodes. Figure2.4 [7] illustrates the difference between stimulated and spontaneous emission mechanisms 

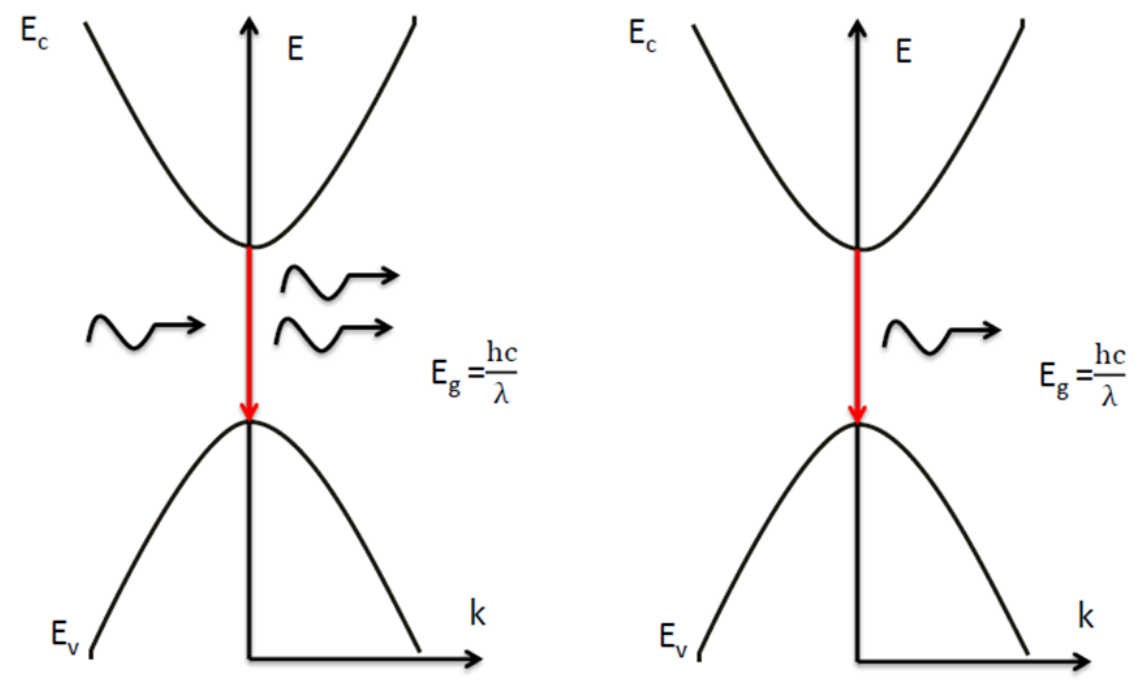

Figure 2.4: Stimulated and Spontaneous Emission Mechanisms [7].

\subsection{Principles of $\mathrm{p}-\mathrm{n}$ junction diode}

The p-n junction diode forms the elemental building block of various solid state devices like, Bipolar Junction Transistors (BJT's), Field Effect Transistors FETs, LEDs, solar cells etc, and are formed by bringing p-type and n-type semiconductors having large concentration gradient of free holes and electrons respectively in contact with each other, creating a large concentration gradient of holes from the $\mathrm{p}$ to $\mathrm{n}$ side across the junction and vice-versa. As the carriers try to diffuse through from the regions of high concentration to the low concentration, they leave behind uncompensated oppositely charged mobile ions. The separation of the positive and negative charges creates an electric field that opposes further diffusion of carriers. This electric field generates drift currents of electrons and holes, exactly matching the diffusion current, that results in the equilibrium condition having the net electric current be zero [27].

A potential gradient $\mathrm{V}_{b i}$, developed by the virtue of this electric filed is the built-in 
potential and determines the band bending in the depletion region [28].

$$
V_{b i}=\frac{k_{B} \cdot T}{q} \frac{N_{A} \cdot N_{D}}{n_{i}^{2}}
$$

In equilibrium the diffusion of holes and electrons from $\mathrm{p}$ to n-side causes accumulation of electrons and holes on the either sides respectively.This region consisting of positive and negative charges on the opposite side of the junction is the depletion or the space charge region, and is calculated using the equation [28]:

$$
W=\sqrt{\left|-V_{b i}\right| \frac{2 \cdot \epsilon_{o} \cdot \epsilon_{s}}{d \cdot N_{D}}}
$$

This depletion layer of the p-n junction can be treated a parallel plate capacitor, and on applying a bias voltage $\mathrm{V}_{a}$ can be calculated using [28];

$$
C=\frac{\sqrt{q \cdot \epsilon_{o} \cdot \epsilon_{s} \cdot N_{D}} \cdot A}{\sqrt{V_{b i}-V_{a}}}
$$

\subsubsection{Bias conditions for $\mathrm{p}-\mathrm{n}$ junction}

It has been studied that, under the equilibrium conditions the formation of a potential barrier restricts the flow of any majority carriers and has no conduction current. For, the p-n junction device to start conducting, the barrier height at the junction is to be reduced so that carriers could flow through. This forms the basis of the forward and the reverse bias operation of the $\mathrm{p}-\mathrm{n}$ junction diode. Let us consider a p-n junction diode connected to a voltage source as shown in Figure 1.5. [26]

The device is said to be forward biased when the p-side of the junction goes to the positive terminal of the source and $n$ to the negative terminal of the source, and reverse biased when the $\mathrm{p}$ goes to the negative terminal and $\mathrm{n}$ to the positive terminal.Under any bias conditions voltage $\mathrm{V}_{a}$ applied across the junction, is expected to appear across the depletion or the space charge region, as the resistivity of the neutral region is small and one expects a minimal drop in these regions. An applied forward bias Voltage $V_{a}$, raises the electrostatic potential on the p-side relative to the $\mathrm{n}$-side and lowers the potential barrier from $\mathrm{V}_{b i}$, to a value equivalent to $\mathrm{V}_{b i}-\mathrm{V}_{a}$, while the reverse bias voltage $\left(\mathrm{V}=-\mathrm{V}_{a}\right)$, depresses the electrostatic potential of p-side relative to the n-side thus increasing the potential barrier from $\mathrm{V}_{b i}$ to $\mathrm{V}_{b i}+\mathrm{V}_{a}$. [26] 


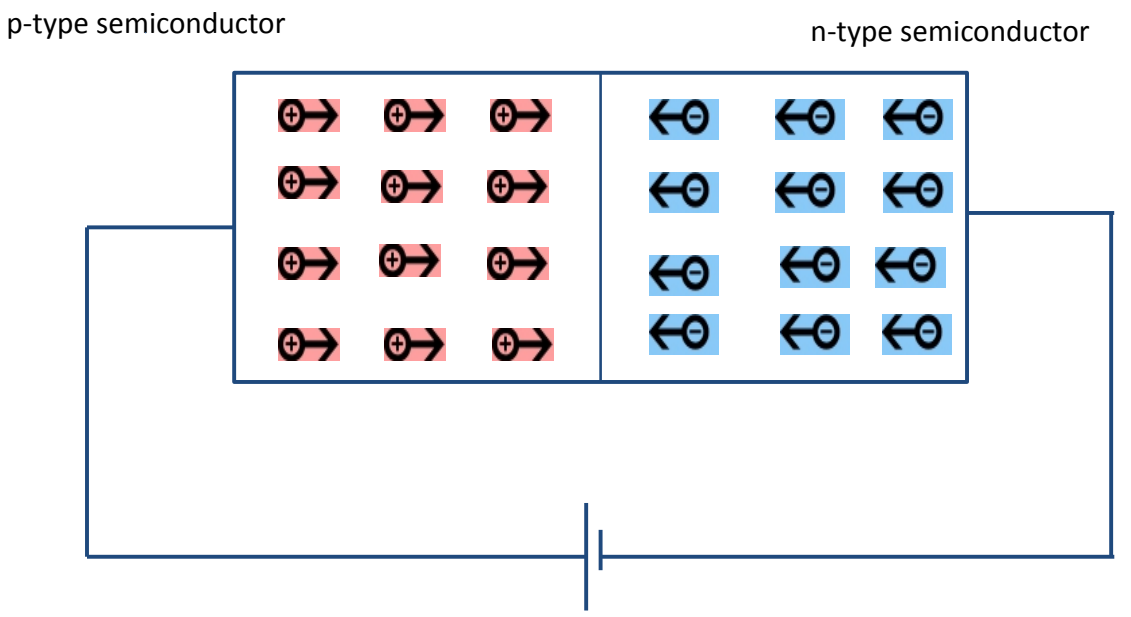

Figure 2.5: P-N junction under bias conditions.

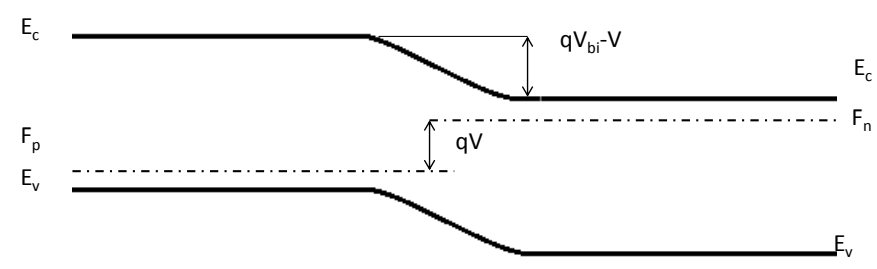

Figure 2.6: Band diagram of p-n junction diode under forward bias conditions.

Thus with the reduction of the potential barrier in the forward biased condition, the drift and diffusion currents are no longer balanced resulting in a net electric current flow. The diffusion current which is due to the majority carrier electrons on the $\mathrm{n}$ side surmounting the potential barrier to diffuse to the $\mathrm{p}$ side and, holes from $\mathrm{p}$ to $\mathrm{n}$ tends to dominate in the forward bias condition due to the reduction of the barrier potential, while in the reverse bias with the increase of the barrier potential electron in the n-side nor holes in the p-side have enough energy to surmount the potential barrier resulting in a very less or no diffusion current. While the drift current which is due to the minority carriers is small due to the number of the minority electrons and holes present.

The Figure below [9] summarizes the forward and the reverse bias condition of a p-n junction device. The two important characteristics of the I-Vs of a p-n junction device are 


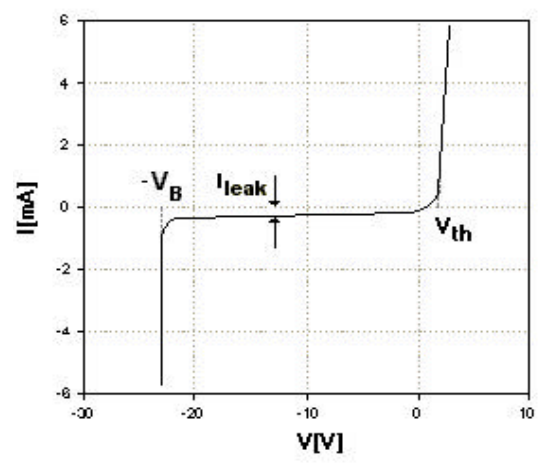

Figure 2.7: I-V characteristics of an ideal p-n junction diode[9].

the threshold voltage $\mathrm{V}_{t h}$ and the reverse leakage current. The threshold voltage is the voltage at which significant conduction from $\mathrm{p}$ - side to the n-side of the junction starts to occur, above which the diode tends to behave as a short circuit with ideally no voltage drop. Under the reverse biased conditions, although the electrons in the n-side and the holes in the p-side of the junction see a huge barrier to cross through for the conduction, the electrons in the p-side see a huge drop in energy to move to the n-side. In other words, when a negative voltage is applied to the p-side of the junction and positive voltage to the n-side of the junction, electrons in the n-type semiconductor see a huge barrier while the electron in the p-type semiconductor can energetically fall down to the n-side, giving rise to a small amount of current called the reverse leakage current and is found to be negative in the I-V characteristics. As the applied reverse voltage increases, the depletion width further increases, causing a further increase in the reverse current that could results in a junction breakdown at higher reverse biases.

The current $\mathrm{I}_{o}$ and the forward currents are related by the Shockley equations. Solving the Shockley equation for the diode, the total net current in $\mathrm{p}-\mathrm{n}$ junction devices is given by the equation [9]:

$$
I=I_{o}\left(\exp \frac{q \cdot V_{a}}{k_{B} \cdot T}-1\right)
$$

Where $\mathrm{I}_{o}$ is the reverse saturation current, $\mathrm{q}$ is the electronic charge, $\mathrm{V}_{a}$ is the applied potential, $\mathrm{k}_{B}$ is the Boltzmann constant and $\mathrm{T}$ is the temperature of the junction. The 
total resistance of the diode can be calculated using the equation [9]:

$$
R_{t}=\frac{V_{a}}{I}=\frac{V_{a}}{I_{o} \cdot \exp \frac{q \cdot V_{a}}{k_{B} \cdot T}}+R_{s}
$$

where $\mathrm{R}_{s}$, is the series resistance. In understanding the $\mathrm{I}-\mathrm{V}$ of the $\mathrm{p}-\mathrm{n}$ junction device it is useful to divide the forward region into two parts, the first part where the exponential term dominates and the second part where the series resistance dominates.

\subsubsection{Non-Idealities in a p-n junction}

The Schokley equation described for the IV characteristics of the p-n junction diode indicates the ideal condition for a p-n junction diode. However, experimental p-n junction diodes are characterized by ideality faction $\eta$, that denoted the deviation from the ideal condition and the I-V relation is governed by, [9]

$$
I=I_{o} \cdot \exp \frac{e V}{\eta k T}
$$

For ideal diodes $\eta$ is expected to be 1 , however more generally for experimental diodes it has values between 1.0 and 2.0. Also, in more reality, a diode may have parasitic resistance like the series resistance and the parallel resistance that should be take into account. A series resistance could be a result of contact resistance, while a parallel resistance could be a result of a bypassing junction to a p-n junction diode. The I-V characteristics of the p-n junction diode can then be modified as [9],

$$
I_{t o t}=I-\frac{V-I R_{s}}{R_{p}}
$$

The series resistance of the diode is usually calculated, by fitting a line equation in the linear part of the IV, from which the slope $\mathrm{dI} / \mathrm{dV}$ is extracted.

\subsection{Metal-Semiconductor Junctions}

The metal-semiconductor junctions serve as regions through which connections are made to an external power supply and play an important role in determining the electrical and 


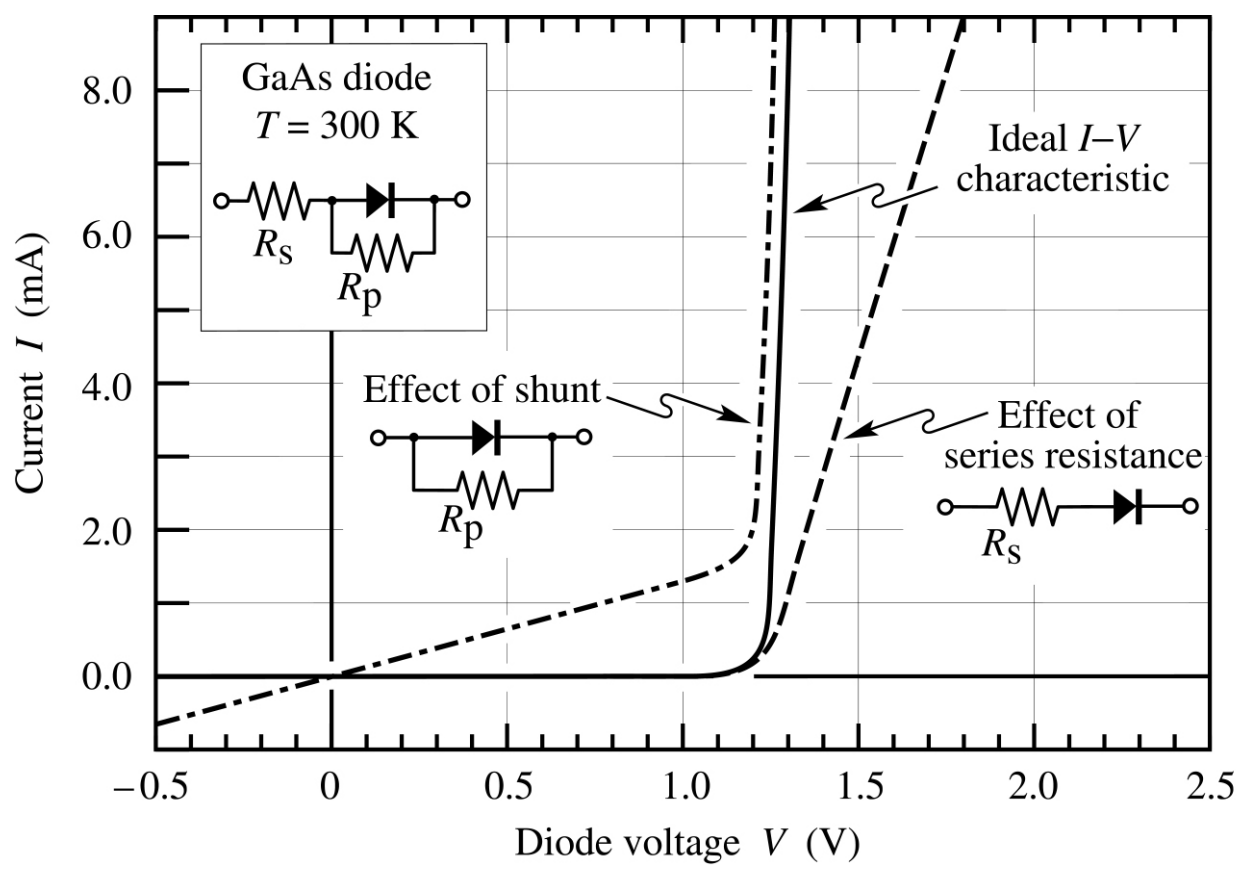

Figure 2.8: I-V characteristics of a p-n junction diode considering the impact of series and parallel resistances $[9]$.

optical characteristics of any solid state device. The metal semiconductor junction or the contacts are classified into different classes the Schottky or the rectifying contacts and the Ohmic contacts depending on the work function difference between the metal and the semiconductor, the work function of a material being the amount of energy required to remove an electron from the fermi level to the vacuum outside the material. Ideally for device applications like LEDs, it is desirable to have ohmic contacts, to have minimum or no voltage drop across the contact.[26]

\subsubsection{Schottky Contacts}

Negative charges brought close to the metal surfaces induce positive charges or image forces, which when acted on by an electric field reduces the work function of the metal to some extent, also called the schottky effect. This forms the basis of schottky contacts. When a metal with work function $\Phi_{m}$ is brought in contact with a semiconductor having a work function $\Phi_{s}$, charge transfer takes place until the fermi levels align at equilibrium. Similar 

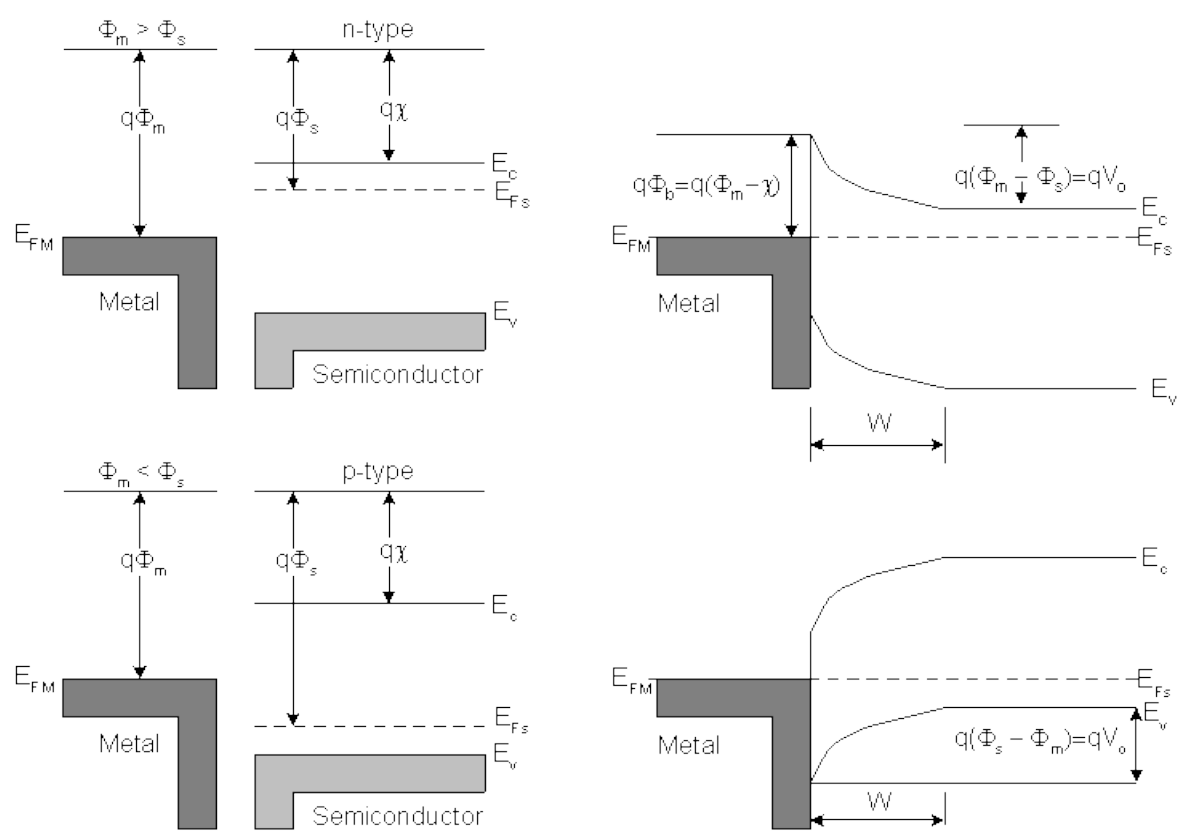

Figure 2.9: Band diagrams of Schottky contacts on p and n-type semiconductors[10].

to the operation of a p-n junction diode, an equilibrium contact potential or barrier height $\mathrm{V}_{b i}$, preventing further diffusion from the semiconductor conduction band into the metal develops at the interface and equals the difference in the work functions of the metal and semiconductor i.e $\Phi_{m}-\Phi_{s}$.

For an electron to get injected from the metal into the semiconductor thus has to overcome the schottky barrier height $\Phi_{B}$, given by $\Phi_{m}-\chi$, where $q \chi$, the electron affinity is the amount of energy required to remove an electron from semiconductor conduction band edge to vacuum. The band diagram in Figure 1.7 gives a representation of a schottky contact to $\mathrm{p}$ and n-semiconductors. The condition for forming a schottky contact on n-type semiconductor is $\Phi_{m}>\Phi_{s}$, and the vice-versa for a p-type semiconductor. The I-V characteristics of a schottky contact are expected to be similar that of a p-n junction, with the fact that it still has a potential barrier [28]. 


\subsubsection{Tunneling contacts}

An alternative contact to the semiconductor devices is the tunneling contact. As, with the case of schottky contacts, tunneling contacts also have a positive barrier, at the metal semiconductor interface however, the semiconductor has a reasonably high doping that the barrier between the metal semiconductor is very thin, thus causing the carriers to tunnel through easily. Tunneling contacts are more common in degenerately doped semiconductors.

\subsubsection{Ohmic Contacts}

Ideal semiconductor contacts are ohmic when the charge induced in the semiconductor in aligning the fermi levels is provided by the majority carriers. The general condition for a metal to form an ohmic contact to an n-type semiconductor is $\Phi_{m}>\Phi_{s}$, for a n-type semiconductor and vice-versa for a p-type semiconductors. The absence of depletion region in these contacts, the potential drop across these junctions is negligible at any bias and therefore, these devices have a linear I-V characteristic in both the bias conditions [28].
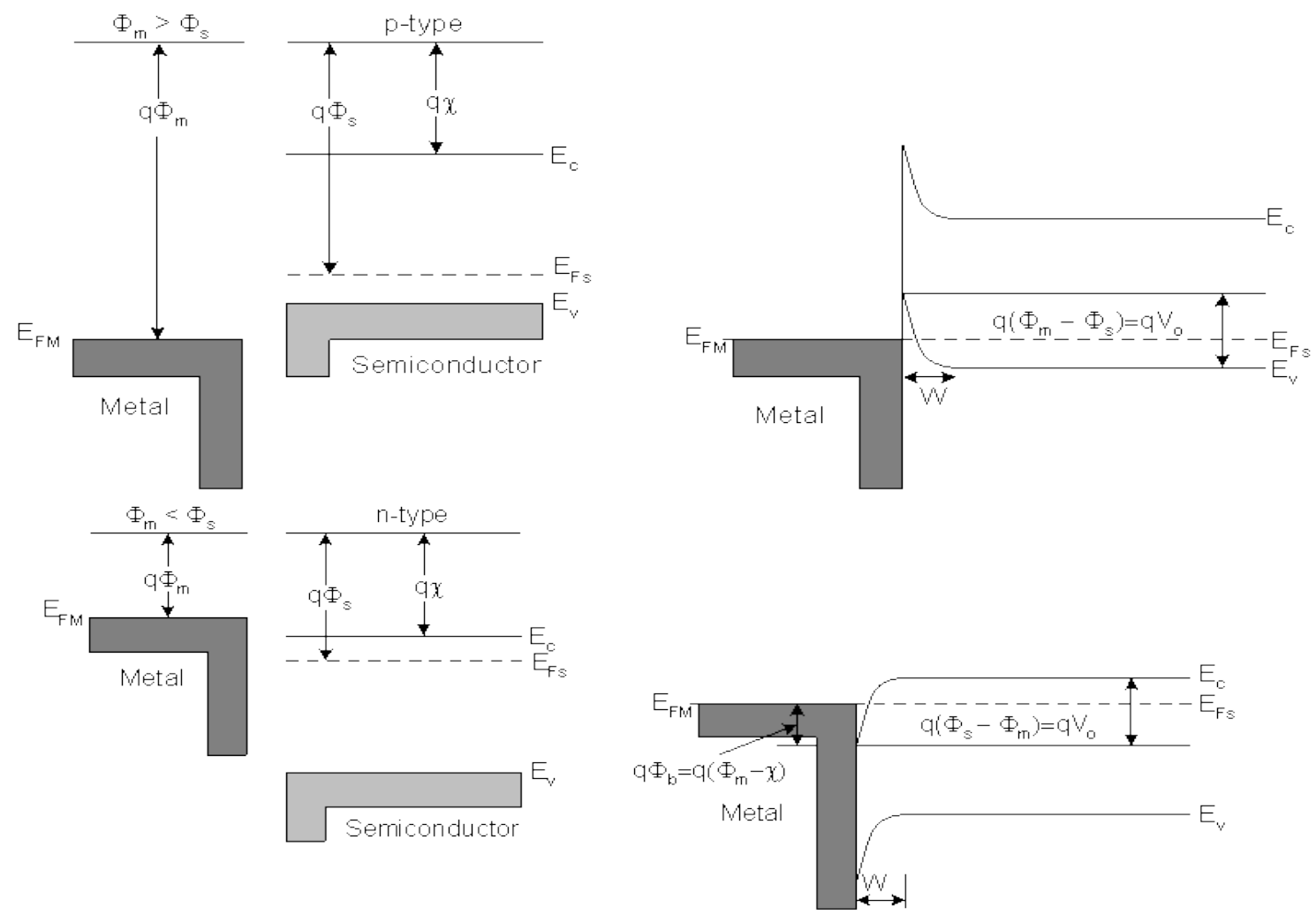

Figure 2.10: Band diagrams of Ohmic contacts on p and n-type semiconductors [10]. 


\subsubsection{Surface effects on metal-semiconductor contacts}

In reality, there could be certain extra allowed states for electrons at the surface of the semiconductor when compared to the bulk [26]. Possible reasons for this could be foreign atoms bonded at the surface, crystal defects, hydroxyl ions etc. The net effect of all these surface states is a density of available electronic states that is not zero. The presence of these surface states could have a significant impact on the contact theory.

\subsection{Quantum Well}

A quantum well is a layered hetereostructure semiconductor device synthesized from two different semiconductors, in which the one thin layer of smaller band gap called well is surrounded by two barriers, layers of wider band gap and the quantum mechanical effects can be controlled. The electrons and holes are both confined to the thin well layer. In LEDs with quantum wells, the carriers are confined to the well region by the two barriers on the either side of the well. As a result, the thickness of the quantum well governs the region of recombination rather than the diffusion length, as is in the case of a p-n junction diode. Diffusion length could be in the range of 1 to 20microns while, quantum well are usually designed to be in the range of 0.1 to 1 micron. As a result, a large concentration of carriers is going to exist in the well layers of the LED, which would result in an increased radiative recombination rate [9].

A bulk semiconductor material assumes a continuum of energy states in the valence band and conduction bands. In the case of a quantum well with reduced thickness, the theory of continuum states no longer holds as the difference between the energy levels is too high. Quantum wells thus have sub bands of energy levels or discrete energy levels.

The discrete energy levels of the quantum well are calculated using the Schrodingers equations:

$$
\frac{-\left(h^{2}\right) d^{2} \Phi \cdot n}{2 \cdot m \cdot d z^{2}}+V(\mathbf{Z}) \cdot \Phi_{n}=E_{n} \cdot \Phi_{n}
$$

The solution to this equation can be obtained either using the infinite well, wherein the barriers on either side of the well are assumed to be infinitely high or the finite well 
conditions. In the case of infinite well method, the energy levels are quadratically spaced, and the wave functions are sine waves, while for the finite well methods, the wave functions are still sine waves inside the well and exponentially decay into the barriers.

A multi quantum well structure (MQW) with a significant penetration of wave function between the quantum wells is called a super lattice. This penetration of wave functions gives rise to mini bands in the structure. In quantum well transition between the conduction and the valence band are allowed only between the states with the same quantum numbers.

\subsection{Light Emitting Diode}

A light emitting diode is an optoelectronic semiconductor $\mathrm{p}-\mathrm{n}$ junction device that emits light on passing electric current through it. The principle components of Light emitting diodes include a back to back sandwich of $\mathrm{p}$ and n-type semiconductor materials forming a p-n junction, characterized by the band gap of the material.
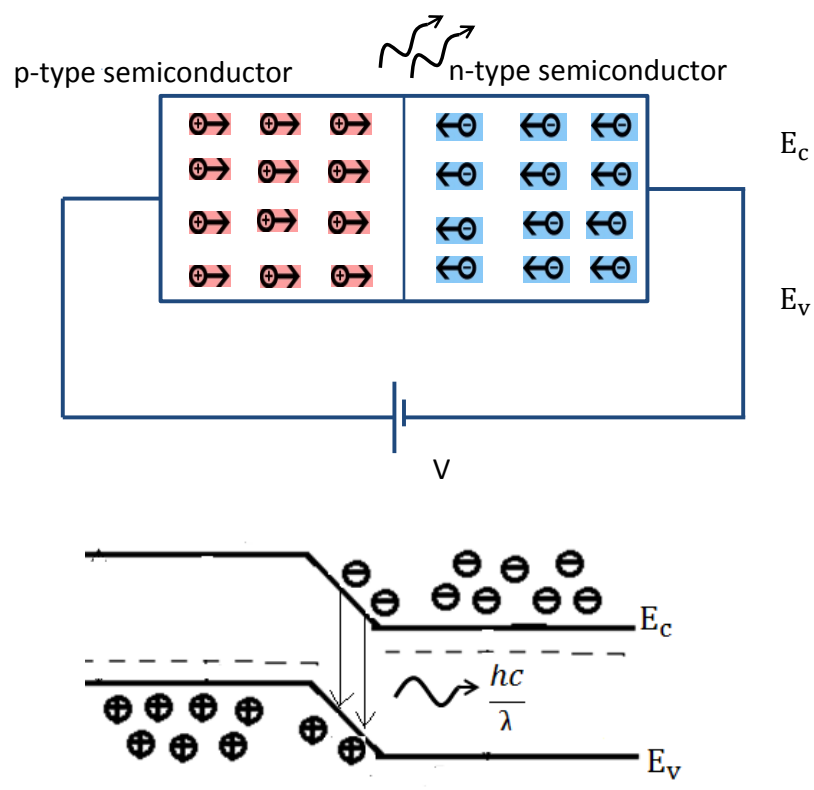

$p$

$\mathrm{n}$

Figure 2.11: Basic light emitting diode.

An LED is a device that converts electrical into optical energy in a two stage process. 
Firstly, the input electrical energy is used to rise the carriers in the semiconductor above their equilibrium state and then these carriers after living their mean life time in the higher energy, tend to fall down giving up their energy as spontaneous emission of photons with, an energy equal to the band gap of the semiconductor. Also, a small fraction of the minority carriers present in the either side of the junction could combine with these majority carriers, and give away their excess energy as phonons. The frequency or the wavelength of the photon emitted depends on the energy difference between the two transition states of the semiconductor and is given by the Plancks relation [28]:

$$
\triangle E=h \cdot \nu=\frac{h c}{\Lambda}
$$

\subsubsection{Choice of Materials}

Different materials have different band gap energies, emitting light from the ultraviolet $(\mathrm{GaN}, 3.4 \mathrm{eV})$ to the infrared $(\mathrm{InSb}, 0.18 \mathrm{eV})$. With the use of ternary and quaternary compounds, the number of available energies could be increased. For example using InGaN as the active layer in LED, photon energies ranging from $1 \mathrm{eV}$ to $3.4 \mathrm{eV}$ could be obtained.

The choice of materials mainly lies between indirect and direct band gap materials. Indirect band gap materials usually have less or relatively no radiative recombinations, as the electrons in the conduction band have different momentum from holes in the valence band causing intermediate recombinations that emit phonons and reduce the internal efficiency of the LED. The peak density of distributions of electrons in the conduction band occurs at an energy slightly above the conduction band minimum. Therefore the band to band transitions emit photons with energy slight greater than their band gap energy, as a result of which some the photons emitted are reabsorbed. However in the case of indirect band gap semiconductors these photons are not reabsorbed due to the involvement of phonons, thus having less radiative efficiency. Another important factor in the choice of material systems is the ability to heavily dope the semiconductor material to achieve $\mathrm{p}$ and n-type materials $[28]$. 


\subsubsection{Recombination Mechanisms in a LED}

As discussed, a light emitting diode is a device that converts electrical energy into optical energy. The supplied electrical energy is used to excite electrons from the valence band to conduction, which upon living for their mean life time tend to fall back and recombine with holes in the valence band to emit the energy. The recombination mechanisms are usually classified as radiative and non-radiative recombinations and are discussed in detail: Radiative recombination and rate equation: In radiative recombination, an electron in the valence band excites into the conduction band on applying an external field, and after living its mean life time falls back to the valence band giving its excess energy as photon.

Any semiconductor has two types of carriers, electrons and holes. Under equilibrium conditions, without any external field, the products of electrons and holes always remain constant [28].

$$
n_{o} \cdot p_{o}=n_{i}^{2}
$$

where, $\mathrm{n}_{o}$ and $\mathrm{p}_{o}$ represent the equilibrium carrier concentrations. Upon applying an external electrical field, and forward biasing the junction; excess carriers are produced in the semiconductor. The electrons in the conduction band now combine with the holes in valence band. The probability that an electron combines with a hole is proportional to the hole concentration, $\propto \mathrm{p}_{o}$. The number of recombinations will also be proportional to the number of electrons. The total recombination rate is thus proportional to the products of electrons and holes $\mathrm{n}_{o} \mathrm{p}_{o}$. Replacing the proportionality constant with Bimolecular recombination coefficient $\mathrm{B}$, gives the biomolecular recombination rate equation [9]:

$$
R_{e q u}=B \cdot n_{o} \cdot p_{o}
$$

Upon applying an external electric field, there are excess carriers induced and the rate equation becomes [9],

$$
R_{\text {net }}=B \cdot\left(n+\left(\triangle n_{o}\right)\right) \cdot\left(p+\left(\triangle p_{o}\right)\right)
$$


(a)

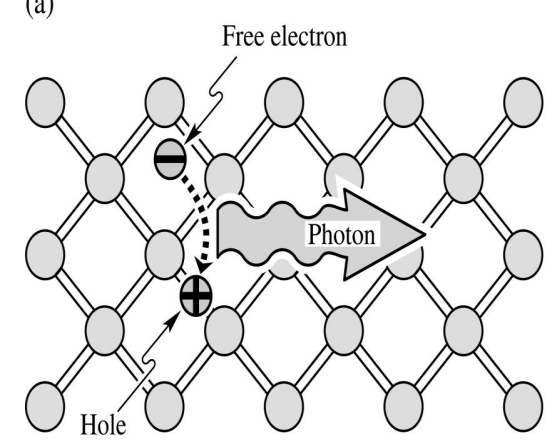

(b)

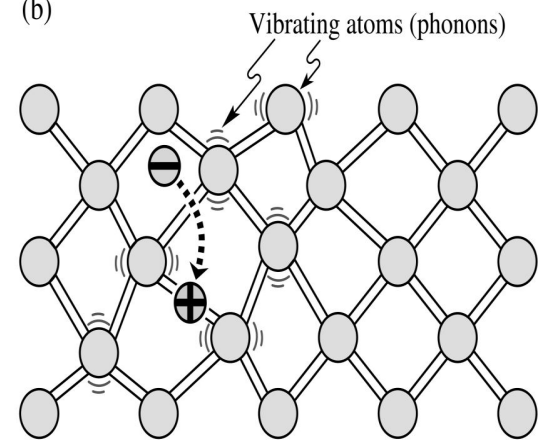

Figure 2.12: Radiative recombination with the emission of a photon and non-radiative recombination creating vibrations in the lattice [9].

Non Radiative Recombination: In non radiative recombinations, the electron energy is converted into vibrational energy of lattice and is thus emitted as phonons.

Some of the causes of the non-radiative recombinations include the quality of the material grown and the fabrication issues, which may give rise to defects, foreign atoms or dislocations. In compound semiconductors these defects could be interstitial levels, vacancies and antisite defects. These defects give rise to intermediate levels that could act as recombination centers, which lie between the forbidden energy band gap of the semiconductor. These centers are therefore called killers [9].

Auger recombination is another kind of non-radiative recombination which becomes important in heavily doped semiconductors. The auger process is a three carrier non-radiative recombination process in which the excess energy released by the formation of an electronhole pair is transferred as kinetic energy to the third free carrier which is raised in energy deep in the respective band. This carrier thermalizes back into bottom of the band [9].

The net recombinations in the LED can thus be represented as,

$$
R_{n e t}=A \cdot n+B \cdot n^{2}+C \cdot n^{3}
$$

The first term represents the Shockley-Read-Hall recombination, second the spontaneous recombination and the last term Auger recombination. 
For light emitting diode applications, it is desirable to have a high ratio of the radiative to non-radiative recombinations. For such high rations, it is necessary to optimize the LED structure to have better carrier confinements and improve the material quality to minimize the defect levels.

\subsubsection{Efficiency}

The different processes involved in the working of a LED are usually classified into three stages, the supply of electrical energy to cause carrier injection into the active layers, known as injection process, the recombination process, in which the carriers excited with the supply of electrical energy tend to transit back, the extraction process associated with the extraction of photons emitted due to the different in the energy levels associated with transition. The overall efficiency of the device is governed by the efficiency of each of these individual processes and is given by [28],

$$
\eta_{0}=\eta_{i n} \cdot \eta_{r} \cdot \eta_{e}
$$

where, $\eta_{o}$ denotes the device efficiency, with $\eta_{i n}$ denoting the efficiency of the injection process, $\eta_{r}$, the efficiency of recombination mechanism, which is usually governed by the ratio of number of radiative recombination to the total number of recombinations, and $\eta_{e}$ denoting the extraction efficiency. The efficiency of the LED is usually characterized by the internal and the external quantum efficiencies. The internal quantum efficiency is governed by the injection and the recombination process and the external quantum efficiency by the extraction process. To have good internal quantum efficiencies, it is necessary to design structures to have better carrier confinement, and material to have less defect, and to have good external quantum efficiencies, it is necessary to have design structures and materials that donot absorb the light emitted and are transparent enough to allow light to be extracted. 


\section{Chapter 3}

\section{Physics and Simulation of Light Emitting Diodes}

Optoelectronic devices like light emitting diodes,lasers etc; brings together electronics and optics into a single device. As discussed in Chapter 2, only some electrons that are loosely bound to the atom are free to move and take part in the conduction process. The conduction process includes, supply of enough energy either in the electrical or the optical form to excite the electron from the valence band to the conduction band, leaving behind a hole which in turn could move in the valence band and cause conduction current to flow to make the net charge zero. The amount of optical or electrical energy required by the valence band electrons to become conduction band electrons is nearly equal to the band gap energy $\mathrm{E}_{g}$ of the characteristic material. After excitation, the conduction band electron falls back to the valence band to attain the stability of the system releasing almost the same energy as photon or phonons. The energy of the emitted photon is related to the wavelength of the emitted light from the relation:

$$
\Lambda_{g}=\frac{h \cdot c}{E_{g}}=\frac{1240 n m}{E_{g}(e V)}
$$

As discussed in Chapter 2; fermi level dictates the highest energy level occupied by an electron in the valence band. The probability of finding an electron with an energy E, is given by the equation 


$$
f(E)=\frac{1}{1+\exp \frac{E-E_{f}}{k_{B} \cdot T}}
$$

where $\mathrm{E}_{F}$ is the fermi energy, $\mathrm{k}_{B}$ is the Boltzmann's constant. In pure or intrinsic semiconductors the fermi level is exactly at the mid band gap energy $\mathrm{E}_{g} / 2$. With the increase of p-type doping the fermi levels gets close to the valence band and on the other hand it gets close to the conduction band with the increase of n-type doping. Also, with increase in temperature or on supply of external energy to the system, electrons are transferred from the valence band to the conduction band, giving rise to the electron and the hole fermi levels which move accordingly.

The actual concentration of the electrons and holes in the conduction or the valence band depends on the density of electron states $\mathrm{D}(e)$ in both the bands. The density of states can be calculated from the equation:

$$
\begin{aligned}
& D_{c}(e)=\frac{1}{\pi^{2}} \cdot\left(\frac{2 \cdot m_{c}}{h^{2}}\right)^{\frac{3}{2}} \cdot \sqrt{E-E_{c}} ; \text { where } E>E_{c} \\
& D_{v}(e)=\frac{1}{\pi^{2}} \cdot\left(\frac{2 \cdot m_{v}}{h^{2}}\right)^{\frac{3}{2}} \cdot \sqrt{E_{v}-E} ; \text { where } E<E_{v}
\end{aligned}
$$

where, $\mathrm{m}_{c}$ and $\mathrm{m}_{v}$ are the effective masses of electrons and holes. The carrier density can be calculated as a function of energy using;

$$
\begin{gathered}
n(E)=D_{c}(E) \cdot f(E) \\
p(E)=D_{v}(E) \cdot[1-f(E)]
\end{gathered}
$$

Assuming the Boltzmanns's distribution holds good, with the assumptuion $\frac{\bmod \left[E_{c}-E_{f}\right]}{k T}$ the above equations as a function of energy bands, gives the total carrier concentration given by;

$$
n=N_{c} \cdot \exp \left(\frac{\left(E_{F}-E_{c}\right)}{K_{B} \cdot T}\right)
$$




$$
\begin{gathered}
p=N_{v} \cdot \exp \left(\frac{\left(E_{v}-E_{F}\right)}{K_{B} \cdot T}\right) \\
f(E)=f_{B}(E)=\exp \left[-\frac{E-E_{f}}{k_{B} \cdot T}\right]
\end{gathered}
$$

The intrinsic carrier concentration $\mathrm{n}_{i}$ can be calculated using the equations:

$$
n_{i}=\sqrt{n p}=\sqrt{N_{c} \cdot N_{v}} \cdot \exp \left(-\frac{E_{g}}{2 K_{B} T}\right)
$$

where $\mathrm{N}_{c}$ and $\mathrm{N}_{v}$ are the effective density of states of the conduction band and the valence band. The above set of equations i.e Boltzmann's distributions hold good only for low carrier concentration i.e $\mathrm{n}<<\mathrm{N}_{c}, \mathrm{p}<<\mathrm{N}_{v}$ ).

However, physically more correct are the fermi statistics which hold good for higher carrier concentrations. For fermi statistics the carrier concentration is given by:

$$
\begin{aligned}
& n \approx N_{c} \cdot F_{\frac{1}{2}} \cdot \exp \left(\frac{\left(E_{F}-E_{c}\right)}{K_{B} \cdot T}\right) \\
& p \approx N_{v} \cdot F_{\frac{1}{2}} \cdot \exp \left(\frac{\left(E_{v}-E_{F}\right)}{K_{B} \cdot T}\right)
\end{aligned}
$$

where $\mathrm{F}_{\frac{1}{2}}$ is the fermi integral of one-half. The above equations can alternatively be written as

$$
\begin{aligned}
& n=\gamma_{n} \cdot N_{c} \cdot F_{\frac{1}{2}} \cdot \exp \left(\frac{\left(E_{F}-E_{c}\right)}{K_{B} \cdot T}\right) \\
& p=\gamma_{p} \cdot N_{v} \cdot F_{\frac{1}{2}} \cdot \exp \left(\frac{\left(E_{v}-E_{F}\right)}{K_{B} \cdot T}\right)
\end{aligned}
$$

where $\gamma_{n}$ and $\gamma_{p}$ are;

$$
\begin{aligned}
& \gamma_{n}=\frac{n}{N_{c}} \exp \left(\frac{E_{c}-E_{F}}{K_{B} T}\right) \\
& \gamma_{p}=\frac{n}{N_{v}} \exp \left(\frac{E_{F}-E_{v}}{K_{B} T}\right)
\end{aligned}
$$




\subsection{Carrier Transport}

As discussed in chapter 2; the drift and diffusion of electrons and holes mainly contribute for the current flow in semiconductors.In this section a brief overview of how the drift and diffusion currents calculations are handled by the simulators will be discussed[28].

\subsubsection{Carrier transport models:Drift and Diffusion}

In general,as discussed in Chapter 2; the drift current is generated by an electric field and is proportional to the conductivity of electrons and holes while, the diffusion current is driven by the concentration gradient of electron and holes [29]. For uniform semiconductors, the total current density of electrons and holes can be written as;

$$
\begin{aligned}
& \overrightarrow{J_{n}}=q \nu_{n} n \vec{E}+q D_{n} \nabla n \\
& \overrightarrow{J_{p}}=q \nu_{p} p \vec{E}+q D_{p} \nabla p
\end{aligned}
$$

q denoting the charge $\vec{E}$ denoting the generated electric field. Now, as an external bias voltage is applied, this equation should be accompanied with a spatial change in current flow and the generation rate $(\mathrm{G})$ or the recombination rate $(\mathrm{R})$ of electron-hole pairs. This relation is known as the continuity equation expressed as:

$$
\begin{aligned}
& q \frac{\partial n}{\partial t}=\nabla \cdot \overrightarrow{J_{n}}-q(R-G) \\
& q \frac{\partial p}{\partial t}=\nabla \cdot \overrightarrow{J_{p}}-q(R-G)
\end{aligned}
$$

For all the above equations to be solved, the electric field itself depends on the charge distribution that include the mobile charge $(\mathrm{n}, \mathrm{p})$ and dopants $\left(\mathrm{n}_{A}, \mathrm{p}_{D}\right)$, and are related by the poisson equation as:

$$
\nabla \cdot\left(\epsilon_{o} \epsilon_{r} \vec{E}\right)=q\left(p-n+p_{D}-n_{A}\right)
$$


Sentaurus Device[30] also includes thermodynamic and hydrodynamic models of transport which are out of the scope of this thesis. To solve for the current densities, the Sentaurus Device uses the conservation law,

$$
\nabla \cdot \vec{J}=0
$$

The current density vector $\vec{J}$, can be return as the curl of current vector potential $\vec{W}$ as

$$
\vec{J}=\nabla \mathbf{x} \vec{W}
$$

The current vector potential $\vec{W}$ has two important properties, the contour lines of $\mathrm{W}$ are the current line of $\vec{J}$ and the difference between the values of $\mathrm{W}$ at any two points is equal to the current flowing between them.

\subsubsection{Boundary conditions for solving continuity equations}

Metals: Metal-semiconductor junctions are usually characterized by the work function difference:

$$
\phi_{M S}=\phi_{M}-\phi_{S}
$$

At contacts, for metal semiconductor junction the dirchlets condition is applied i.e the Fermi potential $\phi_{M}$ is made equal to the applied voltage. Also, Sentaurus Device [30] assumes a metal-semiconductor junction to be ohmic unless otherwise defined as schottky, and at ohmic contacts applies the boundary conditions:

$$
\begin{gathered}
\overrightarrow{J_{M}} \cdot \hat{n}=\vec{J} \cdot \hat{n} \\
\phi=\phi_{M}+\phi_{o} \\
n=n_{0}
\end{gathered}
$$




$$
p=p_{0}
$$

where $\phi_{o}$ is the equilibrium electrostatic potential i.e built in potential and $\mathrm{n}_{o}, \mathrm{p}_{o}$ are the equilibrium concentrations. For n-type semiconductors to have ohmic contacts the metalsemiconductor work function difference $\phi_{M} S<0$ and for schottky contacts, $\phi_{M} S>0$. For schottky contacts, a schottky barrier is formed between metal and semiconductor with a barrier height,

$$
\phi_{B}=\phi_{M}-\chi_{o}
$$

Schottky contacts apart from the drift and diffusion mechanism of transport, involve the thermionic emission and the tunneling mechanisms. The thermionic emission being the thermal heat induced flow of charge carriers and the tunneling being a quantum mechanical phenomenon causes by the wave nature of electron. Electrons arriving at the energy barrier, could either be reflected or penetrated through the barrier, with the penetration depth depending on the barrier height. If the barrier height is thin enough, part of electron penetrated on the other side. The tunneling probability greatly depends on the energy of the electron and barrier height[30]. The schottky contacts are thus modeled using:

$$
\begin{gathered}
\phi=\phi_{F}-\phi_{B}+\frac{k t}{q} \ln \left(\frac{N_{C}}{n_{i, e f f}}\right) \\
\vec{J}_{n} \cdot \hat{n}=q \cdot v_{n}\left(n-n_{o}^{B}\right) \\
\vec{J}_{p} \cdot \hat{n}=q \cdot v_{p}\left(p-p_{o}^{B}\right) \\
n_{o}^{B}=N_{C} \exp \left(\frac{-q \phi_{B}}{K T}\right) \\
p_{o}^{B}=N_{V} \exp \left(\frac{-E_{g, e f f}+q \phi_{B}}{K T}\right)
\end{gathered}
$$

where, $\phi_{F}$ is the fermi potential at the contact that is equal to the applied voltage, $\phi_{B}$ the work function difference between the metal and the electron affinity of the semiconductor, $\mathrm{v}_{n}$ and $\mathrm{v}_{p}$ are the thermionic emission velocities and $\mathrm{n}_{o}{ }^{B}$ and $\mathrm{p}_{o}{ }^{B}$ are the equilibrium carrier densities. 


\subsection{Generation-Recombination Mechanisms in LEDs}

As carriers are exchanged between the conduction and the valence band generationrecombination mechanism processes dominate. The recombination rate $\mathrm{R}$, as studied in the continuity equations included several different mechanisms. The electrons could drop from the conduction band to valence band whereby the electrons need to transfer their excess energy to other particles either as radiative recombination or non-radiative recombination.

\subsubsection{Radiative Recombination}

Radiative recombination is a mechanism in which the electrons in the conduction band fall back to the valence band generating photons.As discussed in Chapter 2; the generation of photons by radiative recombination could be either spontaneous or stimulated. The spontaneous emission rate of photons is characterized by the material coefficient $\mathrm{B}$, and can be written as[30]

$$
R_{\text {spon }}=B\left(n \cdot p-n_{o} \cdot p_{o}\right)
$$

\subsubsection{Auger Recombination}

In Auger recombination, the excess energy associated with the fall of an electron from the conduction band to valence band is transferred to another electron in the valence band or conduction band. The Auger recombination rate is thus written as shown in equation 3.36 to include the density of electrons or holes which could receive the released energy from electron-hole annihilation [28]

$$
R_{n e t}{ }^{A}=\left(C_{n} \cdot n+C_{p} \cdot p\right)\left(n p-n_{i, e f f}^{2}\right)
$$

Auger recombinations become more important in devices with high carrier densities,as the probability of these collisions increase. 


\subsubsection{Shockley-Hall Recombination}

The shockley-hall recombination, takes place due to the deep level traps associated with the device.As electrons from the conduction band tend to fall back to the valence band, there exists some deep level traps with which the electrons could recombine resulting in the emission of a phonon. In Sentaurus Device, the SRH recombination is implemented using:

$$
R_{n e t}^{S R H}=\frac{n p-n_{i, e f f}^{2}}{\tau_{p}\left(n+n_{1}\right)+\tau_{n}\left(p+p_{1}\right)}
$$

where $\tau$ is the life time of either electrons or holes and could be modeled as doping or field dependent and $\mathrm{n}_{i, e f f}$ is the intrinsic carrier concentration.

\subsection{Ray Tracing}

As photons are generated in the device by the electron-hole recombination process, not all the light is extracted from the device because of the optical properties of the devices.A ray of light on a plane surface or materials interface could be reflected, transmitted and absorbed.The ray tracer in Sentaurus Device used a recursive algorithm to trace the light emitted in the device. The sentaurus device starts up with a source ray and maintains a binary tree for the transmission and the reflection of the rays. The transmission or reflection processes occur at interfaces with different refractive indices. The optical output power is then given by summing up the powers of all the transmitted rays that are extracted in a certain window range defined[30].

The simulation of LED devices includes both the electrical and the optical problems As discussed above, the electrical problem involving the Poisson equation, carrier continuity equation and the optical problem involving the generation of photons and extraction of light must be included in the LED simulation.

The solution of the electrical problem yields active region carrier densities. The recombination models studied above are used to compute the spontaneous emission rate, included in the carrier continuity equation to ensure the conservation of particles. 
The spontaneous emission rate is then used to compute the spontaneous emission power which is used by the raytracer to compute the optical intensity. 


\section{Chapter 4}

\section{Modeling the LED}

\subsection{Structure and device parameters used for this study}

\subsubsection{LED structure used for this study}

The basic LED structure used in this study is a planar LED as shown in Figure 4.1 below. The device has been etched through the p-GaN layer and the active layers into the n-GaN

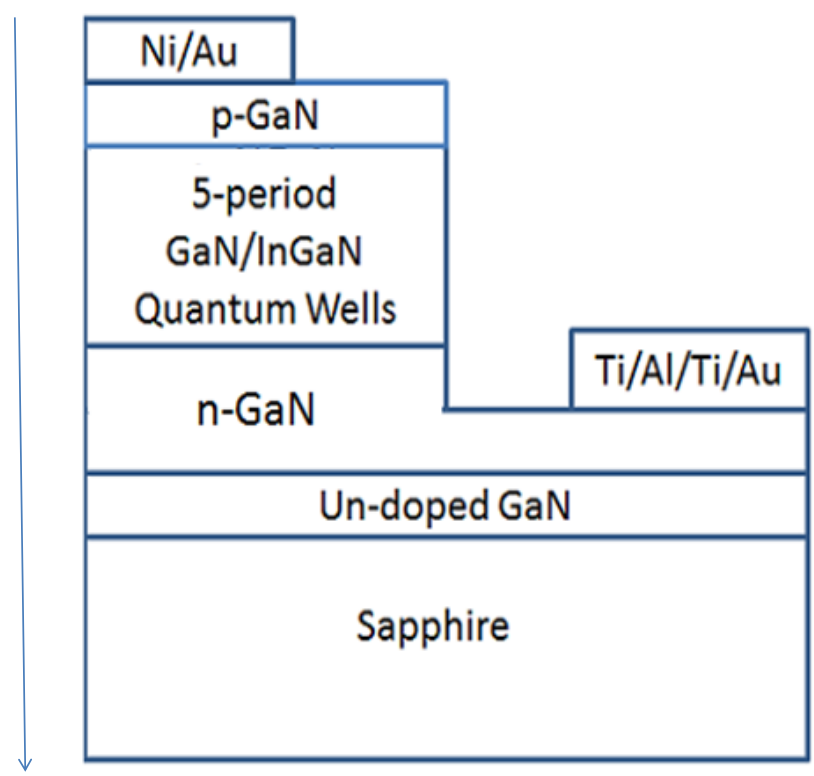

Figure 4.1: LED structure employed for modeling. 


\subsubsection{Material parameters used for this study}

The material parameters have been either experimentally extracted from our MOVPE grown samples or obtained from the literature. The carrier concentrations of different materials have been determined from the Hall effect and CV measurements. The material parameters used in this study are as indicated in the table below:

Table 4.1: Experimentally extracted material parameters

\begin{tabular}{cccc}
\hline Material & Doping $\left(\mathrm{cm}^{-3}\right)$ & Mobility $\left(\mathrm{cm}^{2} /\right.$ V.s $)$ & Thickness $(\mathrm{nm})$ \\
\hline p-GaN & $3 \times 10^{17}$ & 10 & 70 \\
p-AlGaN & $3 \times 10^{17}$ & 10 & 10 \\
GaN barrier & & & 8.8 \\
InGaN active layer & & 200 & 4.8 \\
n-GaN & $1 \times 10^{18}$ & & 1500 \\
un-doped GaN buffer layer & & & 500 \\
sapphire & & & 1000 \\
\hline
\end{tabular}

Table 4.2: Material parameters from literature

\begin{tabular}{ccc}
\hline Material & Electron Affinity $(\mathrm{eV})$ & Band Gap $(\mathrm{eV})$ \\
\hline p-GaN & 3.4 & 3.4 \\
GaN barrier & 3.4 & 3.4 \\
$\mathrm{In}_{x} \mathrm{Ga}_{1-x} \mathrm{~N}$ active layer; $\mathrm{x}=0.16$ & 3.956 & 2.787 \\
n-GaN & 3.4 & 3.4 \\
un-doped GaN buffer layer & 3.4 & 3.4 \\
sapphire & 0.5 & 6.1 \\
\hline
\end{tabular}

Electron affinity of GaN has been reported over the range of $3.2+/-0.2 \mathrm{eV}, 3.5+/-$ $0.1 \mathrm{eV}, 4.1+/-0.1 \mathrm{eV}$, in the literature with $4.1 \mathrm{eV}$ being the upper limit [31][32][33]. It has been indicated that an electron affinity of $3.4 \mathrm{eV}$ obtained from the vacuum referred binding energy model matches well with the experimental results, and is thus used in the development of the TCAD model. The material parameters of InGaN have been interpolated from the parameters of $\mathrm{InN}$ considering the band gap of $\mathrm{InN}$ to be $1.0 \mathrm{eV}$ and electron affinity to be $5.1 \mathrm{eV}$. The developed model takes into account only the radiative recombinations and the non radiative recombinations have not been included due to the difficulties associated with the extraction of the deep level traps present in the material.

The band gap of $\operatorname{In}_{x} \mathrm{Ga}_{1-x} \mathrm{~N}$ has been calculated using; 


$$
E_{g}\left(\operatorname{In}_{x} G a_{1-x} N\right)=x * E_{g}(\operatorname{In} N)+(1-x) * E_{g}(G a N)-b * x *(1-x)
$$

where $\mathrm{E}_{g}(\operatorname{InN})=1.0 \mathrm{eV}$; $\mathrm{b}$ is the bowing factor and is equal to 1.7.

The electron affinity of $\operatorname{In}_{x} \mathrm{Ga}_{1-x} \mathrm{~N}$ has been calculated using

$$
\chi\left(\operatorname{In}_{x} G a_{1-x} N\right)=x * \chi(\operatorname{In} N)+(1-x) * \chi(G a N)
$$

The effective masses of electrons and holes have been considered from the literature to be 0.2 and 1.1. The radiative recombination coefficient of the active layer has been taken to be $2 * 10^{17} \mathrm{~cm}^{-} 3$.

\subsection{Preliminary Modeling Results}

TCAD model utilizing the drift diffusion model has been developed for the structure discussed. The model couples the poisson's equation and the continuity equation using newton's iteration method.

\subsubsection{Band Structure}

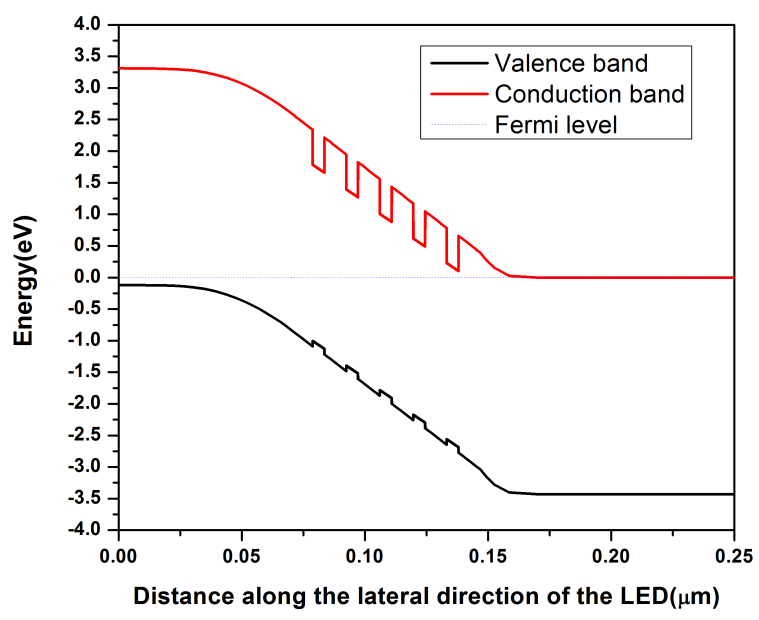

Figure 4.2: Band diagram of the LED device plotted along the vertical direction of the LED. 
The band diagram of such a structure is as indicated in the Figure 4.2. Light emitting diodes fabricated with this kind of a structure couldn't light up or were emitting relatively less optical power. Possible reason for this could be the fact that,the electrons having higher mobility than that of holes, are likely overflowing into the p-GaN layer due to insufficient holes in the active layers, and the number of possible radiative recombinations in quantum wells are as a result relatively less. To overcome this problem, light emitting diodes with p-AlGaN layer as an electron blocking layers (EBL) have been proposed and are compared to the structure with out an EBL. The device structure is now as indicated in the Figure 4.3:
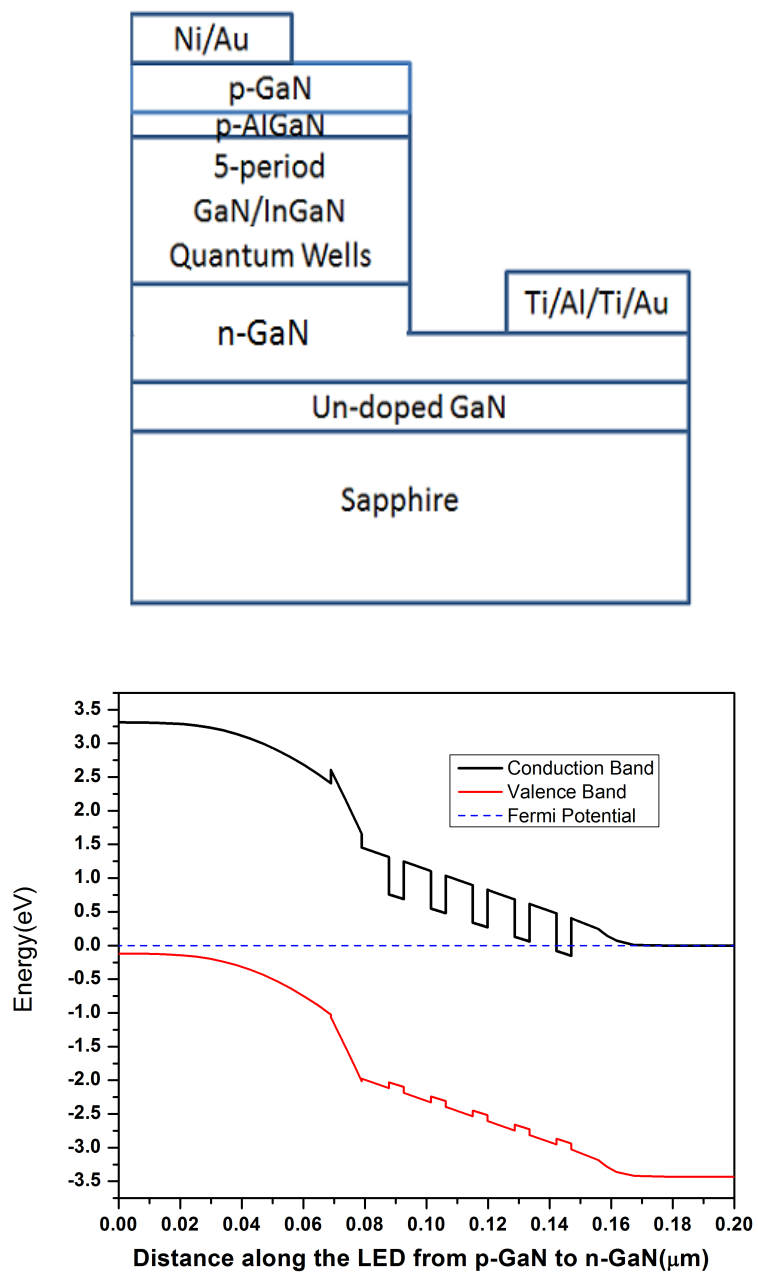

Figure 4.3: Structure and band diagram of the LED device without the p-AlGaN layer plotted along the lateral direction of LED. 
The electron affinity and the band gap of AlGaN have been interpolated in the manner similar to that of InGaN and the molefraction of $\mathrm{Al}$ is taken to be 0.1 and a thickness of $10 \mathrm{~nm}$. The band diagram of such a structure is as indicated in the Figure 4.3.

It is evident from the band diagram of this structure that, a p-AlGaN EBL layer would have a bump in the conduction band acting as the barrier for the electrons flowing from the active layer to the p-GaN. Thus there could relatively be more electrons and holes confined in such a structure and is evident from the carrier distribution plots and radiative recombination rate plots of both the structures as shown below in Figures 4.4 and 4.5.
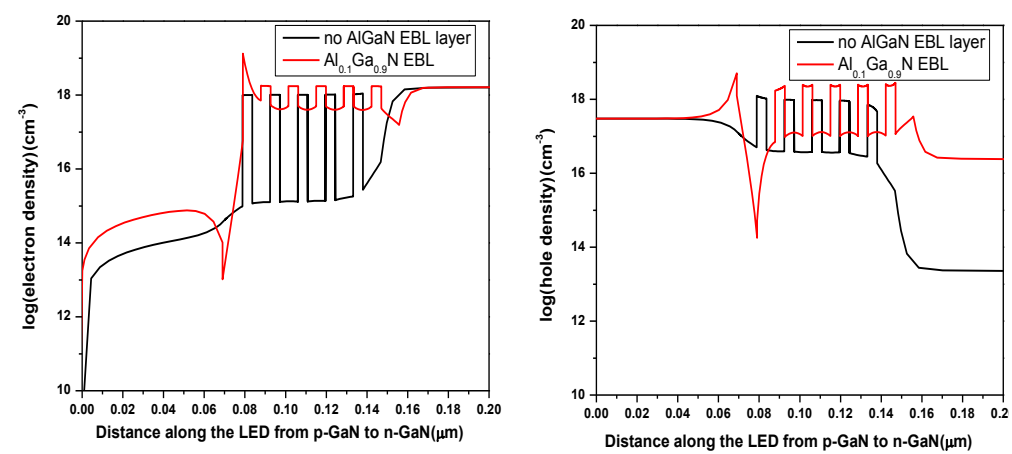

Figure 4.4: Electron and hole distribution along the LED from p-GaN to n-GaN.

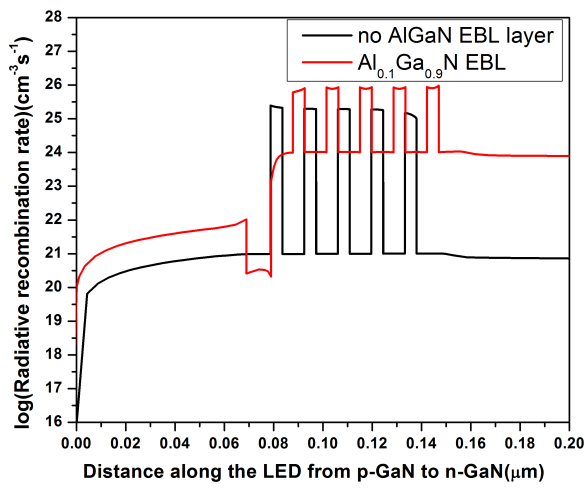

Figure 4.5: Radiative recombination along the LED from p-GaN to n-GaN.

The plots plotted on a log scale indicate that there is an improvement in the confinement of electrons and holes to the quantum wells thus resulting in an increase in the radiative 
recombination rate in the quantum wells.

\subsection{Validation of the developed model}

The developed model has been validated by simulating different devices. The Figure below indicates the mask used to fabricate LEDs:

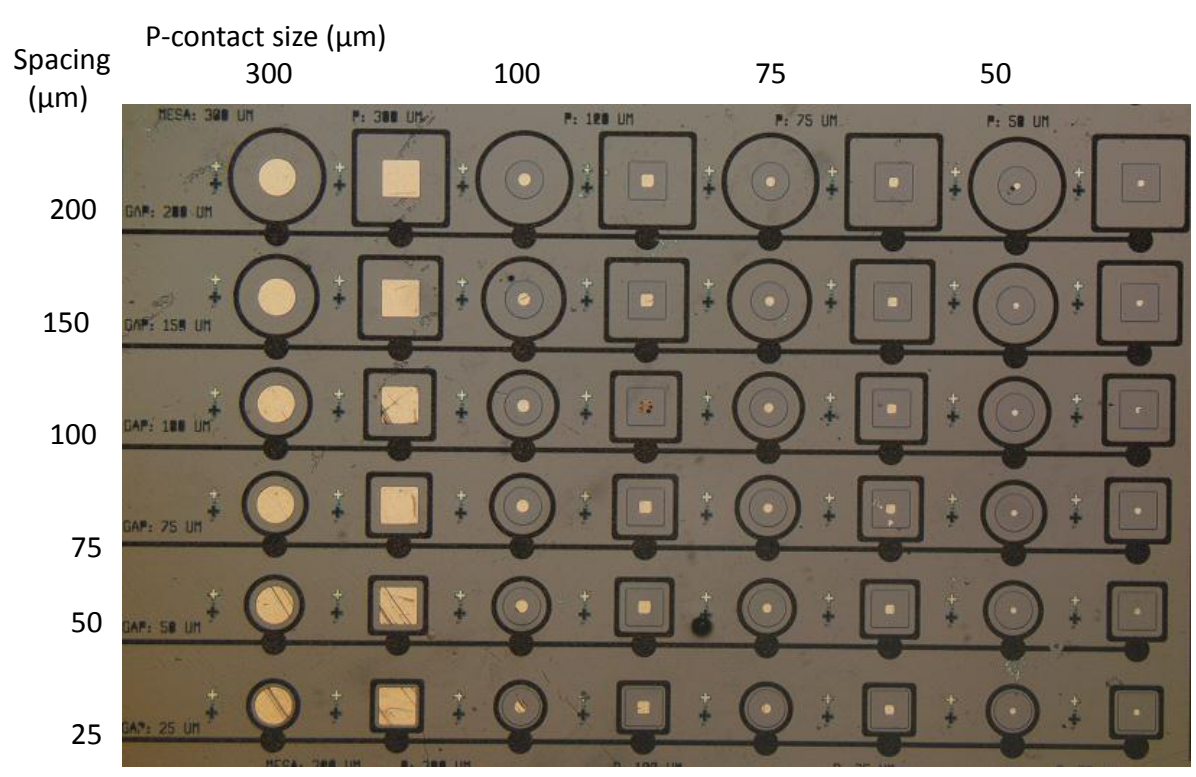

Figure 4.6: Mask used to fabricate the LEDs.

The bright gold color spot, at the center of each device on the mask indicates p-contact, surrounded by a thin grey line indicating the p-mesa, further surrounded by a black color border indicating the n-contact. The mask has in total 48 devices, left to right indicating devices that having different p-contact sizes and top to bottom indicating devices with different spacings between p-mesa and n-contact. The legend $\mathrm{p}$ on the top of the mask indicates p-contact size and the legend gap to the left of the mask indicating spacing between the p-mesa and n-contact.

These different devices have been simulated keeping the material parameters constant, same and varying the spacing between the contacts. The current-voltage (IV) characteristics 
of the different devices simulated are plotted with the experimental data and are as indicated in the plots in Figure 4.7
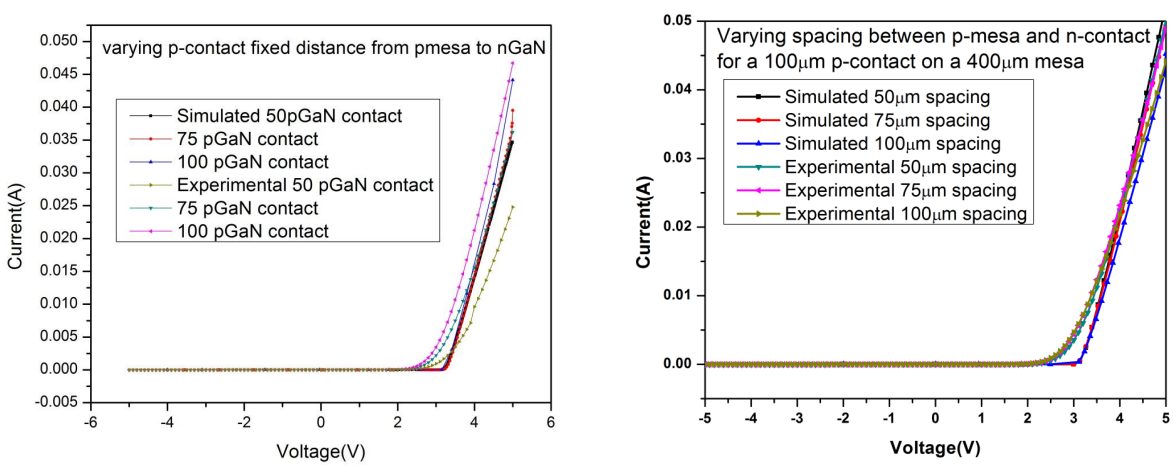

Figure 4.7: Simulated and experimental IV plots for different LEDs (a) varying the p-contact size with the spacing between p-mesa and n-contact being 25 microns (b) varying the spacing between p-mesa and n-contact with the p-contact size 100microns.

The series resistance for different devices are indicated in the Figure 4.8.

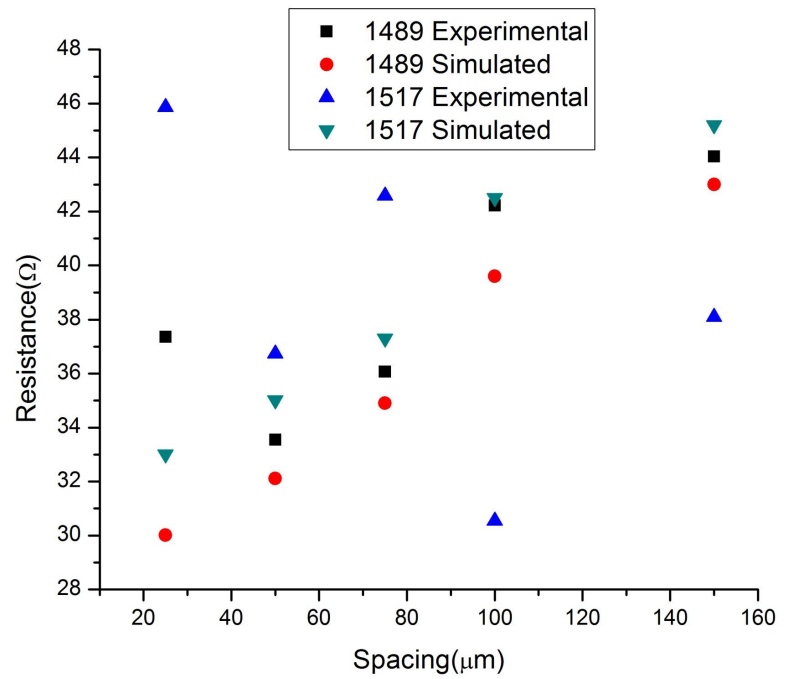

Figure 4.8: Resistance plots comparison for different devices with a p-contact size of 100microns, varying the space between p-mesa and n-contacts.

The results from the model developed, had certain deviations from the experimental 
model which could be attributed to the fact that the polarization charges between the GaN/InGaN interface have not been considered, and that the electron affinity and the band gap have been taken from the literature and are not experimentally extracted from our MOVPE grown sample due to certain resource limitations. Series resistance for these devices have been extracted from the IV plots, by calculating $\mathrm{dI} / \mathrm{dV}$, and extracting the maximum values and the resistance to be the inverse of this value [9]. Another possible reason, could be that there could be some experimental error associated with the extraction of parameters from the MOVPE grown samples.

\subsection{Analysis of effectiveness of AlGaN layer for carrier confinement}

The effectiveness of AlGaN was studied varying the composition and thickness to have the optimal set of values for better confinements.

\subsubsection{Composition Variation}

The composition of AlN in AlGaN was varied from 0.05,0.1 to 0.2 and the band diagrams are as indicated in the Figures below:

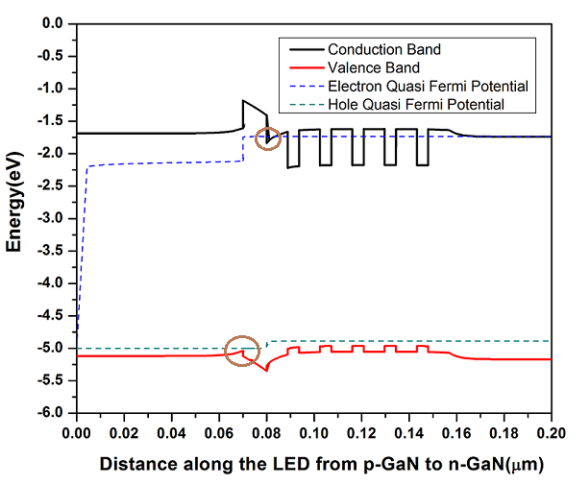

Figure 4.9: Band diagram for $\mathrm{x}=0.2$ in $\mathrm{Al}_{x} \mathrm{Ga}_{1-x} \mathrm{~N}$. 

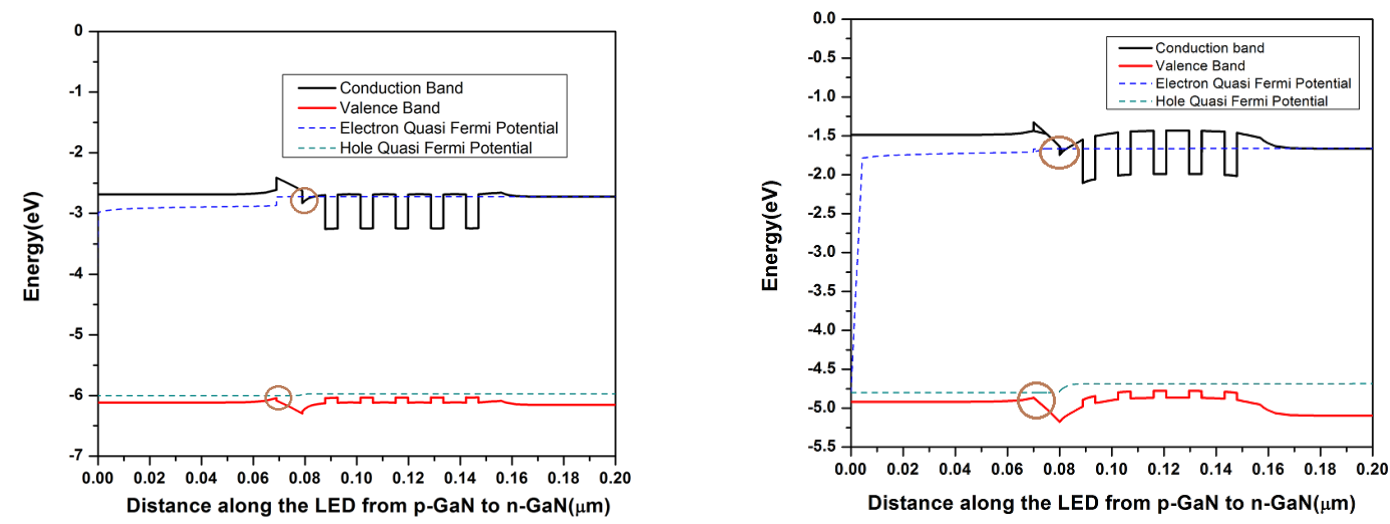

Figure 4.10: Band diagram for $\mathrm{x}=0.2 \mathrm{x}=0.1$ and $\mathrm{x}=0.05$ in $\mathrm{Al}_{x} \mathrm{Ga}_{1-x} \mathrm{~N}$.

The p-AlGaN layer indicates spikes and notches in the conduction band and valence band that vary with the $\mathrm{Al}$ composition. The spike indicated in the valence band could help in confining the electrons to the active layer by acting as a barrier. However, in the conduction band it could be observed that, holes could get accumulated at p-GaN/AlGaN interface and could see a barrier at the p-AlGaN/GaN barrier interface influencing the flow of holes into the quantum well.
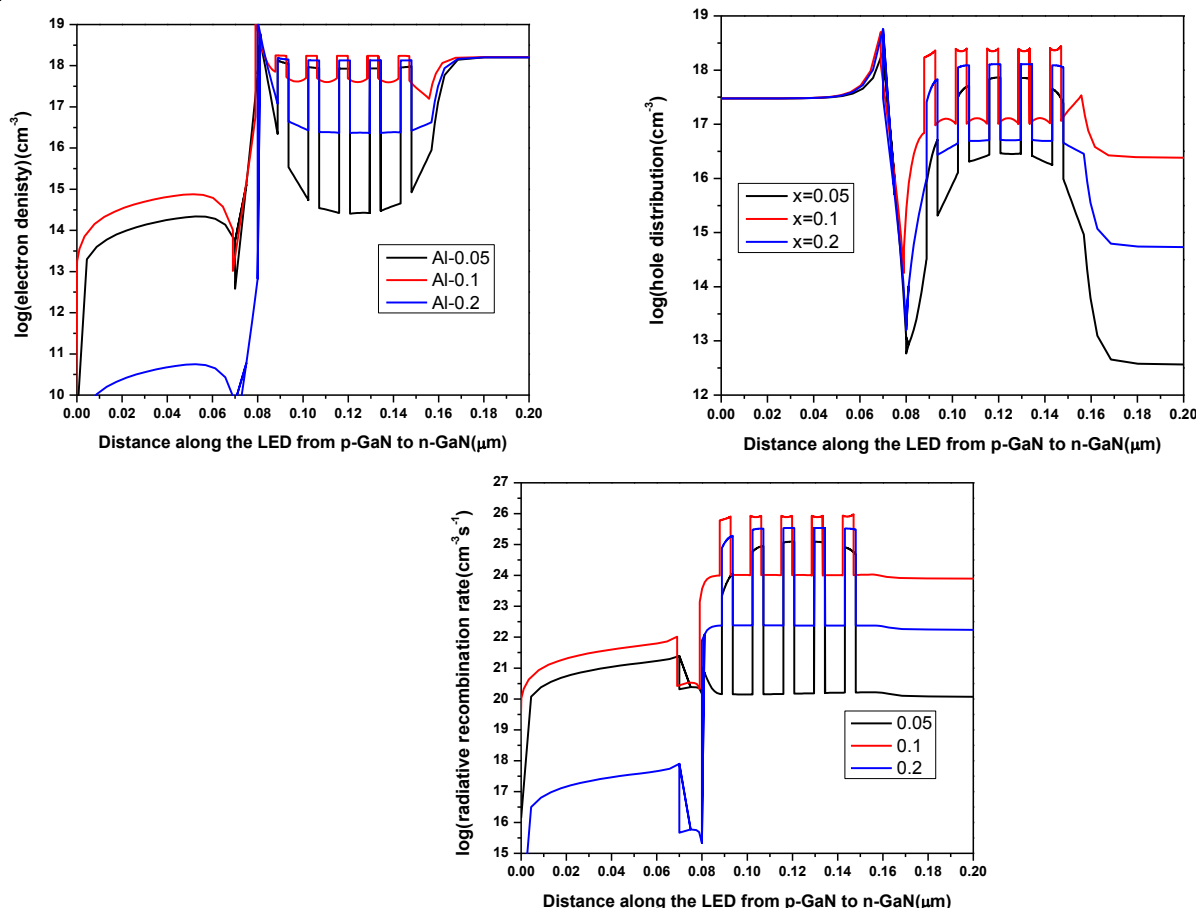

Figure 4.11: Electron distribution, hole distribution and radiative recombination rates in the $\mathrm{LED}$ with varying $\mathrm{Al}$ composition. 
The carrier distribution for these three different devices are as indicated in the Figure 4.11. It is evident from the distribution plots that, the electron distribution is relatively the same in the quantum wells, while the hole concentration, changes with the composition of AlGaN. Results indicate that for $\mathrm{x}=0.05$, hole distribution is relatively non uniform which possibly could be due to the barrier height between the p-AlGaN and GaN barrier while $\mathrm{x}=0.1$ and 0.2 have relatively uniform distribution of holes and radiative recombination rate while $\mathrm{x}=0.1$ is one order of magnitude more than $\mathrm{x}=0.2$. The quasi fermi potentials of the band diagram also indicate that there are more number of electron - hole confinements in the active layer in the case of $\mathrm{x}=0.1$ over $\mathrm{x}=0.05$ or $\mathrm{x}=0.2$.

\subsection{Influence of thickness}

From, the results shown above the composition of $\mathrm{Al}$ in $\mathrm{AlGaN}$ was taken to be 0.1 and simulations were carried out varying the thickness of the electron blocking layer. The band diagrams of the simulated structure for thickness of $10 \mathrm{~nm}, 20 \mathrm{~nm}$ and $40 \mathrm{~nm}$ are as indicated in the Figures below:

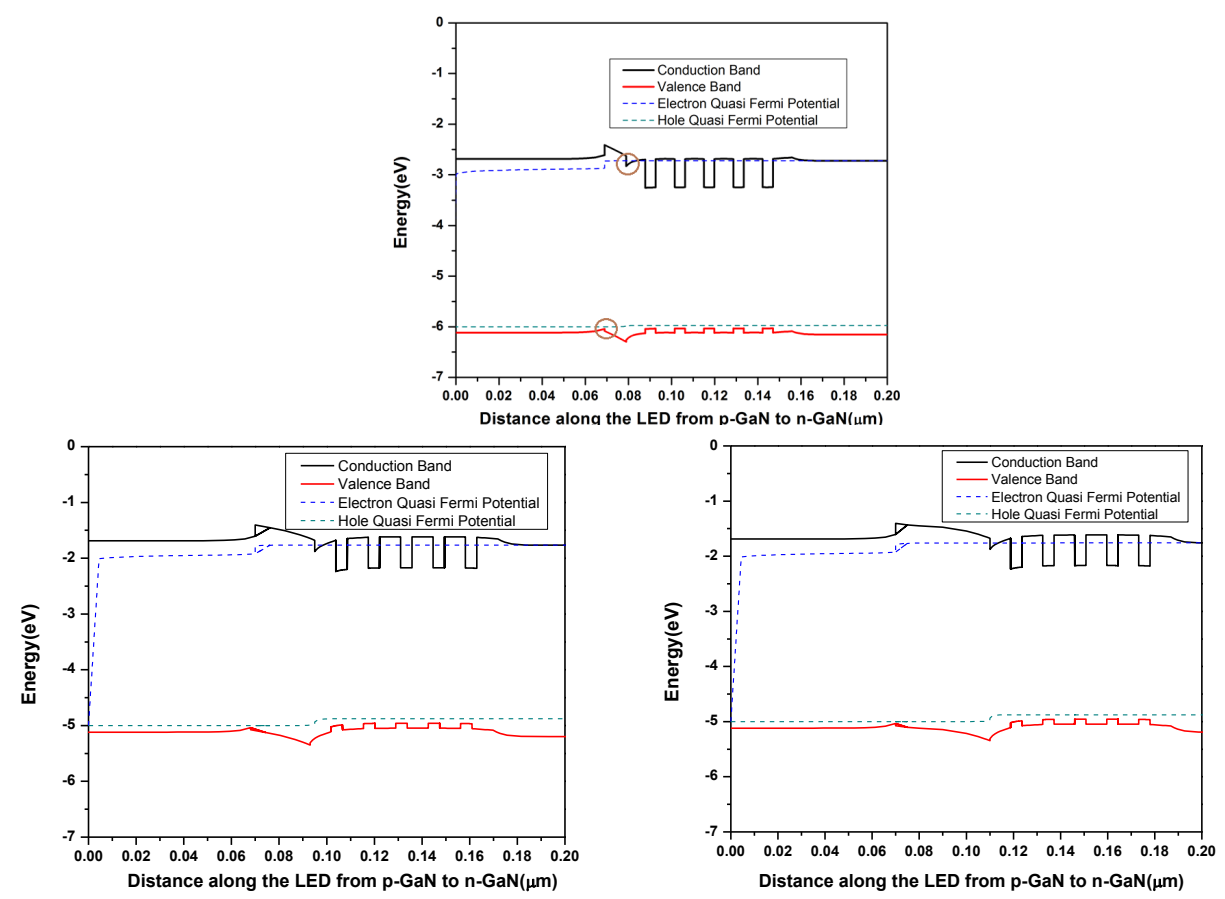

Figure 4.12: Band diagram for the LED structure varying the thickness of the EBL to be $10 \mathrm{~nm}, 20 \mathrm{~nm}$ and $40 \mathrm{~nm}$. 
The carrier distribution and radiative recombination plots are as shown in Figure 4.13 below. From the Figure, it is conclusive that all the quantum wells have relatively the same distribution of electrons but non-uniformly distributed holes. This could be explained by the fact that conduction band and the valence band of all the three structures have the same kind of spikes and notches, however, the hole crowding that could occur at the p-GaN/AlGaN interface could potentially easily tunnel through or thermionically into the thin p-AlGaN layer of $10 \mathrm{~nm}$ while would take relatively more time for higher thicknesses. The radiative recombination plots, as expected are governed by the hole distribution. From this study, a $\mathrm{p}$-AlGaN electron blocking layer of $\mathrm{x}=0.1$ and thickness $=10 \mathrm{~nm}$ is used for our devices.
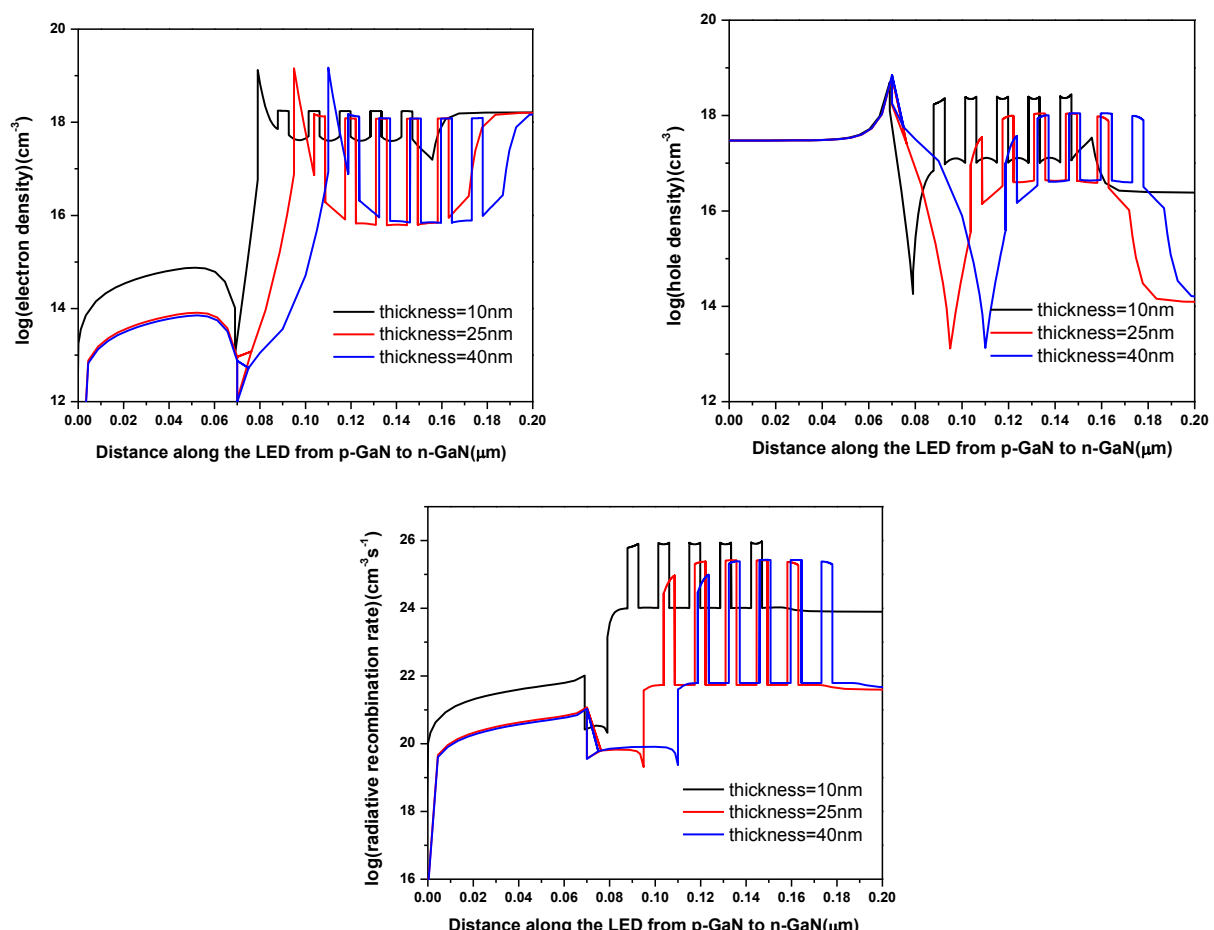

Figure 4.13: Electron, hole and radiative recombination rate distributions in the quantum wells. 


\section{Chapter 5}

\section{Design analysis}

\subsection{Drawbacks of the previous structure}

The planar LEDs with the structure discussed in chapter 4 has certain limitations. One such limitation is the current crowding problem. Having known that the n-GaN is approximately two order of magnitude more than the p-GaN, the current from the p-contact tries to flow in to the n-contact through path just under the contact edge rather than spreading through the entire mesa. This could better understood by the graphs indicated below in Figure 5.1.

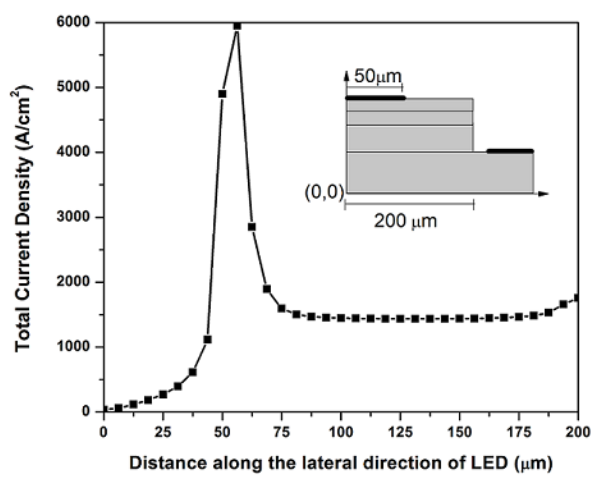

(a)

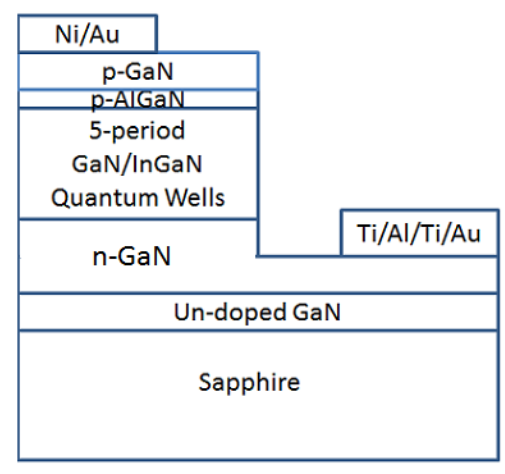

(b)

Figure 5.1: Current density distribution plot (a) along the lateral direction of the LED in the first quantum well for the structure shown (b). 
The graph in the Figure 5.1 shows current current crowding at the edge of a 50 microns contact on a 200 mesa. This current crowding could result in associated unwanted heating effects and a reduction of the extraction efficiency of the device given the fact that the $\mathrm{Ni} / \mathrm{Au}$ contact is semi-transparent in nature. Proper analysis of the design parameters is thus necessary to study the impact of different layers and bring up design changes in the structure to have a more uniform current spreading. If the current density across the contact edge could be taken as $\mathrm{J}_{o}$, the current distribution $\mathrm{J}(\mathrm{x})$ in the mesa can be represented as

$$
J(x)=J_{o} \cdot \exp (-\alpha \cdot x)
$$

\subsubsection{Analysis of the structure}

In this study PSPICE has been used to study the impact of the resistance of each layer on the current spreading. A resistance based analytical model has been developed for each layer of the LED, however the series resistances in the LEDs have not been taken into account. The model has been validated in PSPICE by applying a current I across the $\mathrm{p}$ and $\mathrm{n}$ materials, and studying if the distribution of the current is following the same path as expected from the analytical model. The series resistance of each individual layer is calculated from the conductivity and thickness of the layer using the equations:

$$
\text { Conductivity }=(\text { carrier concentration }) *(\text { mobility }) *(\text { charge of an electron })
$$

Having known the conductivity, the resistivity could be written as:

$$
\text { resistivity, } \rho=\frac{1}{\text { conductivity }}
$$

The series resistance of each layer could then be extracted using the relation:

$$
\text { Resistance, } R=\frac{\rho}{t}
$$


where $\mathrm{t}$ denotes the $\rho$ and $\mathrm{t}$ denote the resistivity and thickness of the corresponding layer.

The quantum wells could each be modeled as a p-n junction diode. The ideality factor of these diodes could be extracted from the I versus V plot of the LED.

The simplified resistance model used for this study is as indicated in the Figure 5.2.

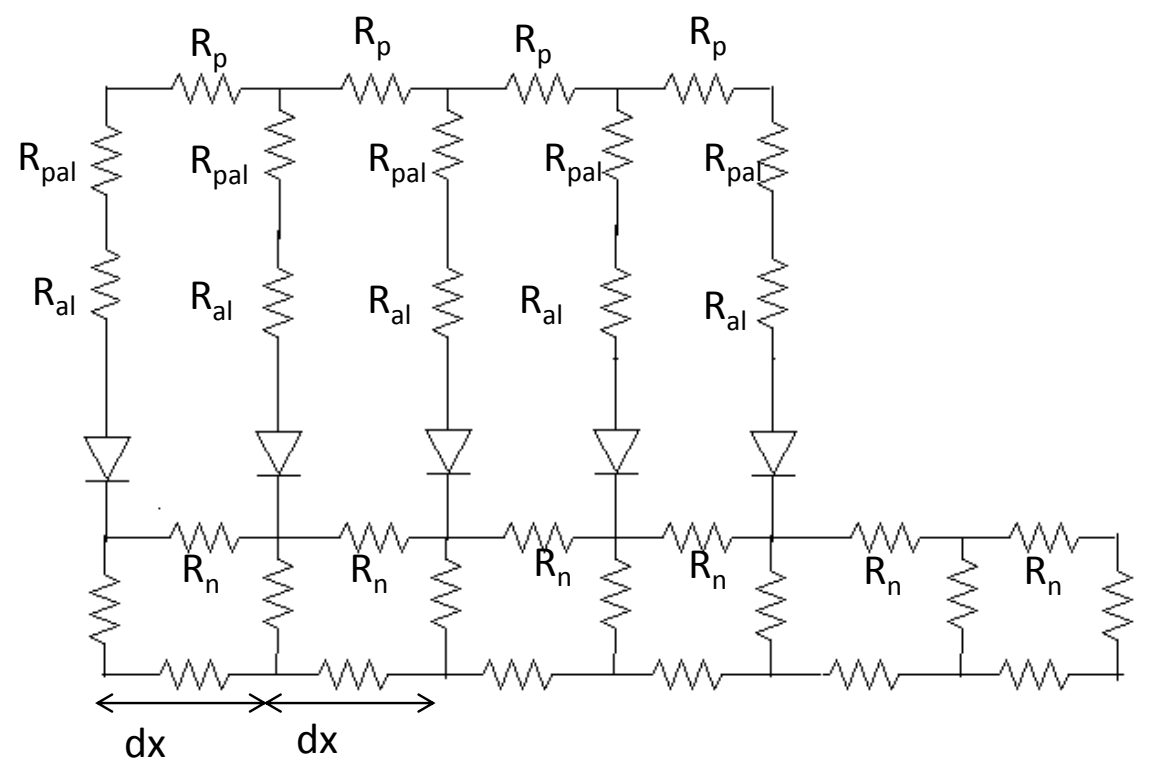

Figure 5.2: Resistance model representation of the LED.

The notation in this plot indicates, $\mathrm{R}_{p}$ to be the sheet resistance of $\mathrm{p}-\mathrm{GaN} \mathrm{R}_{\text {pal }}$ to be the resistance at the $\mathrm{p}-\mathrm{GaN}, \mathrm{p}-\mathrm{AlGaN}$ interface, $\mathrm{R}_{a l}$ to be the sheet resistance of $\mathrm{p}-\mathrm{AlGaN}$ and $\mathrm{R}_{n}$ be the sheet resistance of $n-G a N$. The active layers of the LED are represented using the diodes.

To make the analysis simpler, a simple resistance model as shown in Figure 5.3 is considered

Let up suppose that a current I, is entering the structure at the contact edge. In such a structure the current entering into the junction could take either path 1 or path 2 . The current flowing through path 1 and path 2 is given by,

$$
I_{\text {path } 1}=\frac{I \cdot\left(R_{v}+R_{n}\right)}{2 R_{v}+R_{n}+R_{p}}
$$




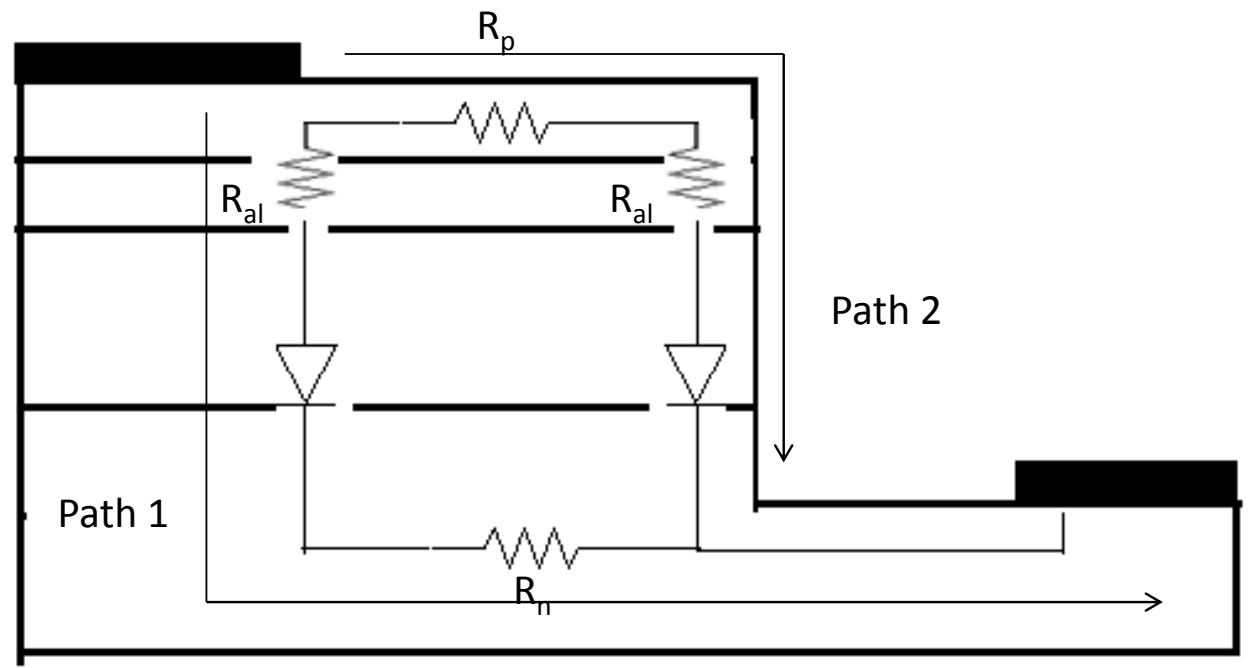

Figure 5.3: Simplified resistance model representation of the LED.

The current flowing through path 2 is given by,

$$
I_{\text {path } 2}=\frac{I \cdot\left(R_{v}+R_{p}\right)}{2 R_{v}+R_{n}+R_{p}}
$$

where $\mathrm{R}_{v}$ denotes the resistance of the $\mathrm{p}-\mathrm{AlGaN}$ and the diode. To have a uniform current through the active layers, the current flowing through path 1 should be same as the current flowing through path 2 , which can be written as

$$
R_{p}=R_{n}
$$

To have a uniform current spreading in such a device it is necessary to have equal current flowing through the active layer. Three different conditions have been taken to study the impact of $\mathrm{p}-\mathrm{GaN}$ an $\mathrm{n}-\mathrm{GaN}$ on the current distribution. The conditions include $\mathrm{R}_{p}>>\mathrm{R}_{n}$, $\mathrm{R}_{p}<<\mathrm{R}_{n}, \mathrm{R}_{p} \approx \mathrm{R}_{n}$ 

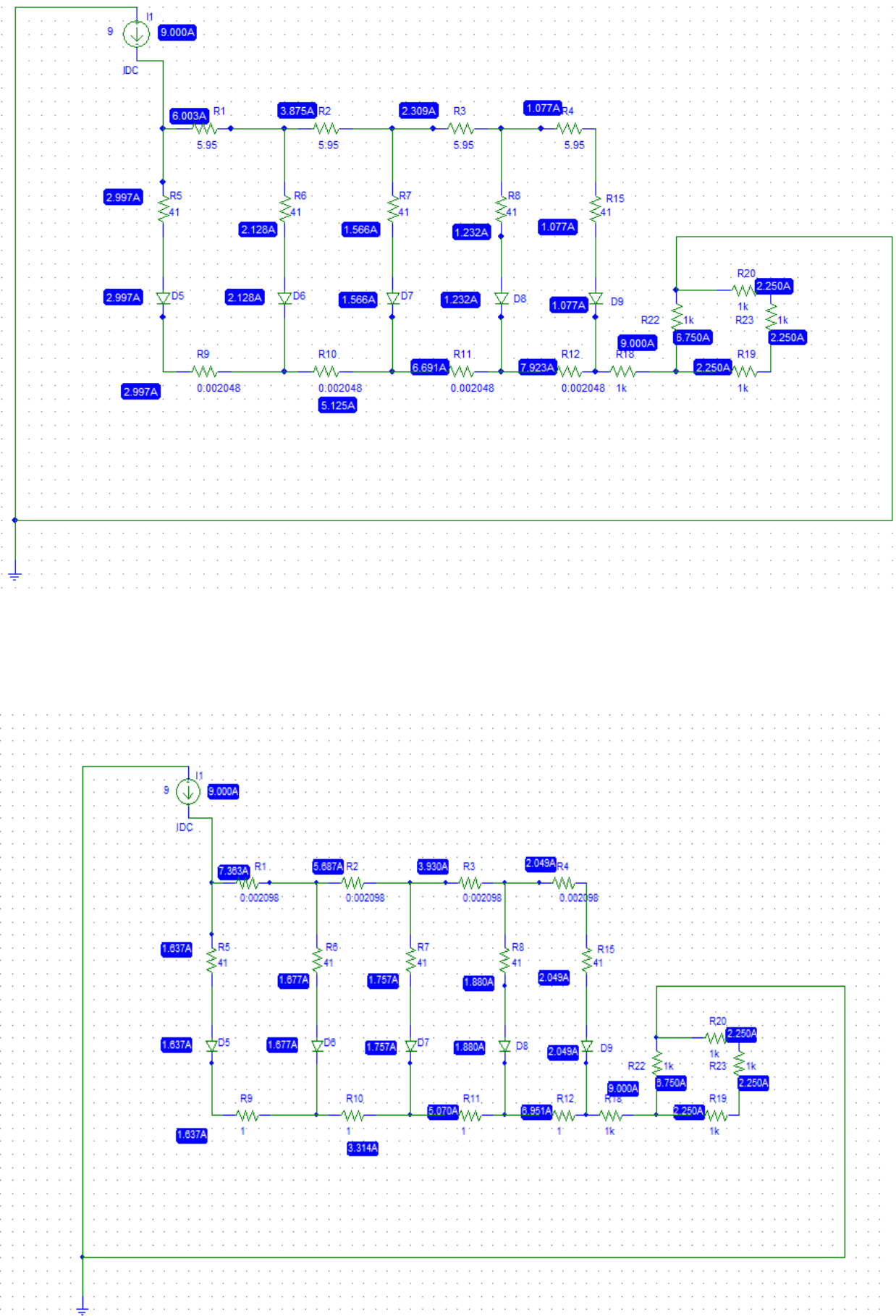

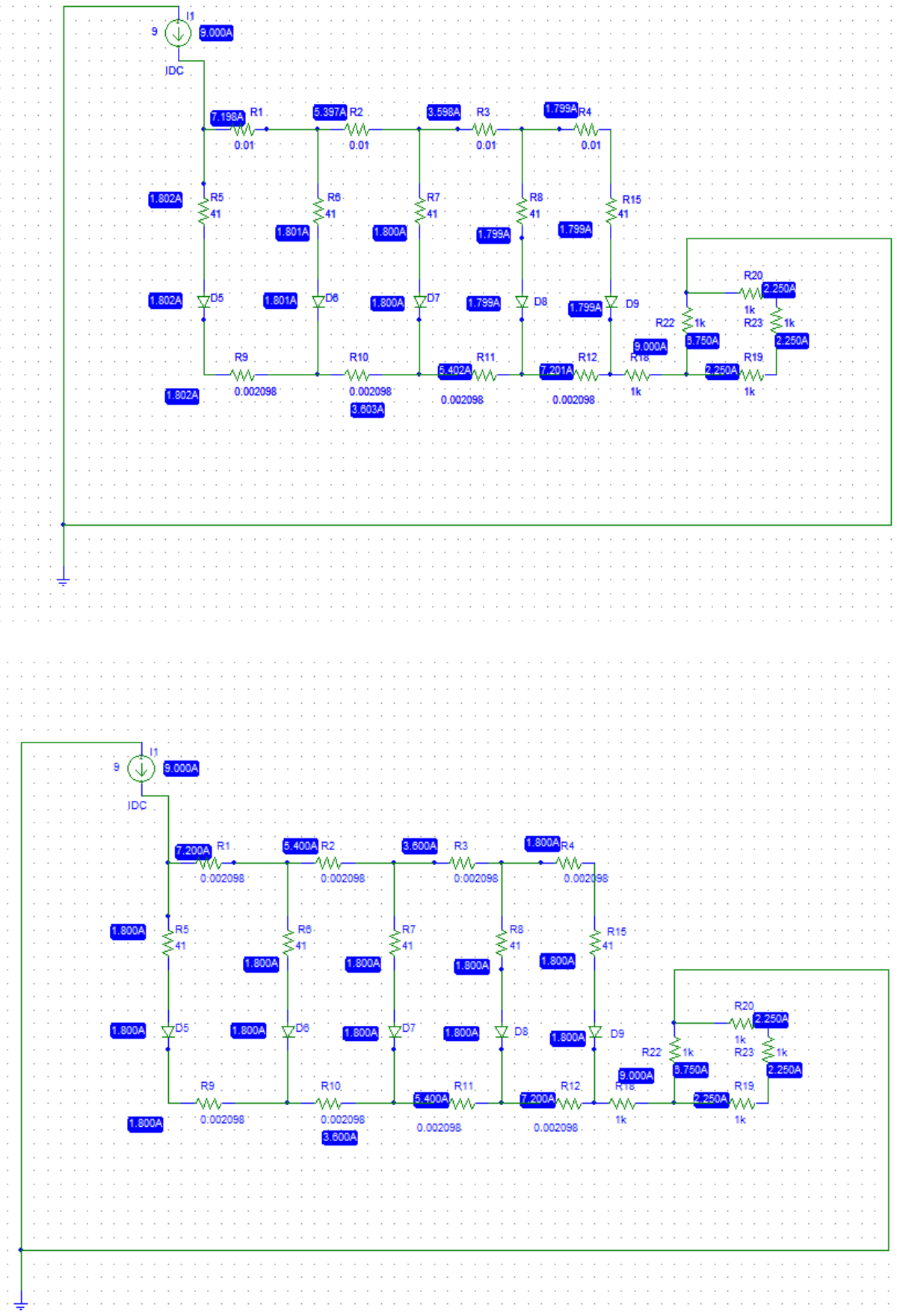

Figure 5.4: PSPICE modeling of the resistance model for the condition $\mathrm{R}_{p}>\mathrm{R}_{n}, \mathrm{R}_{n}>\mathrm{R}_{p}$, $\mathrm{R}_{n} \approx \mathrm{R}_{p}, \mathrm{R}_{n}=\mathrm{R}_{p}$. 
Thus the ratio of the resistance between $\mathrm{p}-\mathrm{GaN}$ and n-GaN thus act as a deciding factor for current crowding.

- If $R_{p}<R_{n}$, current crowds at the edge of the p-mesa.

- If $R_{n}<R_{p}$, current crowds at the edge of the p-contact.

- If $R_{n} \approx R_{p}$, current approaches to be uniform.

- If $R_{n}=R_{p}$, current is uniform in the active layers.

\subsection{Design changes to p-GaN}

From the PSPICE model, it is indicated that to have a better spreading the resistance of $\mathrm{p}-\mathrm{GaN}$ is to be comparable to the resistance of $\mathrm{n}-\mathrm{GaN}$ which is similar to

$$
\frac{\rho_{p}}{t_{p}}=\frac{\rho_{n}}{t_{n}}
$$

It is well known that the conductivity of $\mathrm{n}-\mathrm{GaN}$ is approximately three orders of magnitude more than the conductivity of $\mathrm{p}-\mathrm{GaN}$, and the thickness of $\mathrm{n}-\mathrm{GaN}$ is approx two times that of n-GaN, thus causing the current to crowd at the mesa edge. An increase in the thickness of $\mathrm{p}-\mathrm{GaN}$ could thus result in an increase in both the internal and the external extraction efficiencies, due to the fact that it would have more number of holes and a minute increase in the thickness would result in a better current spreading.
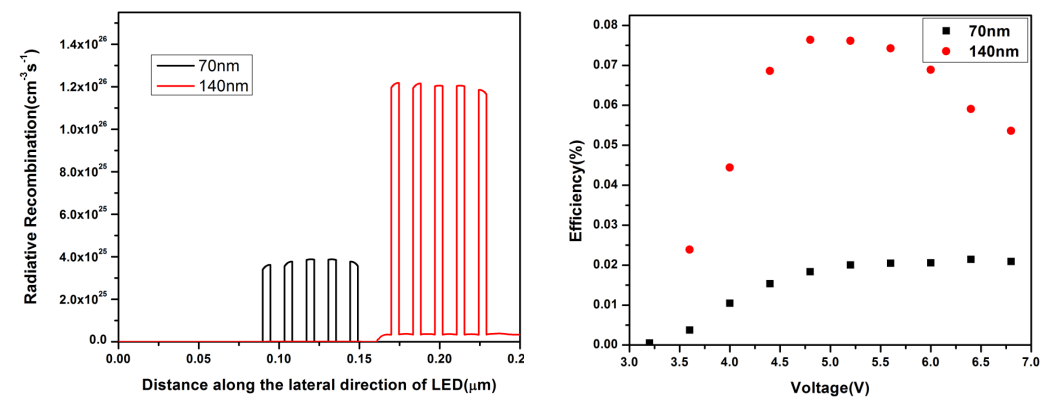

Figure 5.5: Illustration of increase in the efficiency of $140 \mathrm{~nm}$ thick p-GaN over $70 \mathrm{~nm}$ thick p-GaN. 
It is evident that when both the resistance are made comparable a uniform current spreading could be achieved. However, it is experimentally challenging to grow such highly conductive p-GaN layer and alternative changes such as use of transparent conducting oxides have to be studied, and is dealt in the next chapter. 


\section{Chapter 6}

\section{Potential of AZO as transparent conducting contact to $\mathrm{p}-\mathrm{GaN}$}

\subsection{Design of AZO as transparent contact to p-GaN}

As discussed in chapter 5 , the regular Ni/Au contacts on LEDs showed current crowding at the contact edge, thus motivating the need for study of alternative transparent contacts. $\mathrm{Ni} / \mathrm{Au}$ contacts being semitransparent in nature with transparency around 60-75\% in visible and lower than $60 \%$ in UV region[34], have the probability of blocking the light emitted at the contact edge. Having known these drawbacks associated with the regular Ni/Au contact design of transparent conducting oxide contact to $\mathrm{p}-\mathrm{GaN}$ is gaining its research interest.

Transparent conducting oxides like indium tin oxide (ITO) aluminium alloyed zinc oxide (AZO) etc are synthesized from semiconductor materials indium oxide (I2O3) and zinc oxide $(\mathrm{ZnO})$ via chemical doping of tin $(\mathrm{Sn} 4+)$ ions for indium $(\mathrm{In} 3+)$ and aluminium $(\mathrm{Al} 3+)$ for zinc $(\mathrm{Zn} 2+)$ ions, respectively. Degenerate doping of these semiconductor materials with their respective dopants creates a transition from semiconducting state to metallic state, thus making the material highly conducting yet sustaining its fundamental band gap property, and remaining optically transparent[35].

Indium tin oxide (ITO) has been widely investigated by several groups as a challenging material for transparent contacts for optoelectronic device applications and transmittance reports of nearly $85 \%$ and resistivity's of the order of $10-4 \Omega \mathrm{cm}$ have been demonstrated 
[36][37][38]. ITO's insufficient thermal stability for use as transparent contacts in high power LEDs, its toxicity and the high cost involved in its manufacture has generated interest in the use of alternative materials for transparent contacts.[39][40]

$\mathrm{ZnO}$ has been studied as an alternative material for transparent contacts and several reports have been published of gallium doped zinc oxide and aluminium doped zinc oxide with electrical and optical properties similar to ITO[41][42]. Although AZO could be used as a promising material for its application as a transparent contact to LEDs, little research has been carried out given that AZO is expected to have a work-function less than 4.1eV[43] and is expected to form a Schottky contact with p-GaN[44] which has a work-function of around $6.6 \mathrm{eV}[21]$.

In this study, the AZO/Ni interface has been studied. The carrier concentration of AZO was take to be $5 \times 10^{20} \mathrm{~cm}^{3}$. Simulation results indicate that with such high doping of AZO an increase in the carrier concentration of $\mathrm{p}$-GaN from $10^{17} \mathrm{~cm}^{3}$ to $10^{18} \mathrm{~cm}^{3}$ for p-GAN the $\mathrm{AZO} / \mathrm{p}-\mathrm{GaN}$ junction formed had a depletion width of around $65 \mathrm{~nm}$ as depicted in figure 6.1 below.

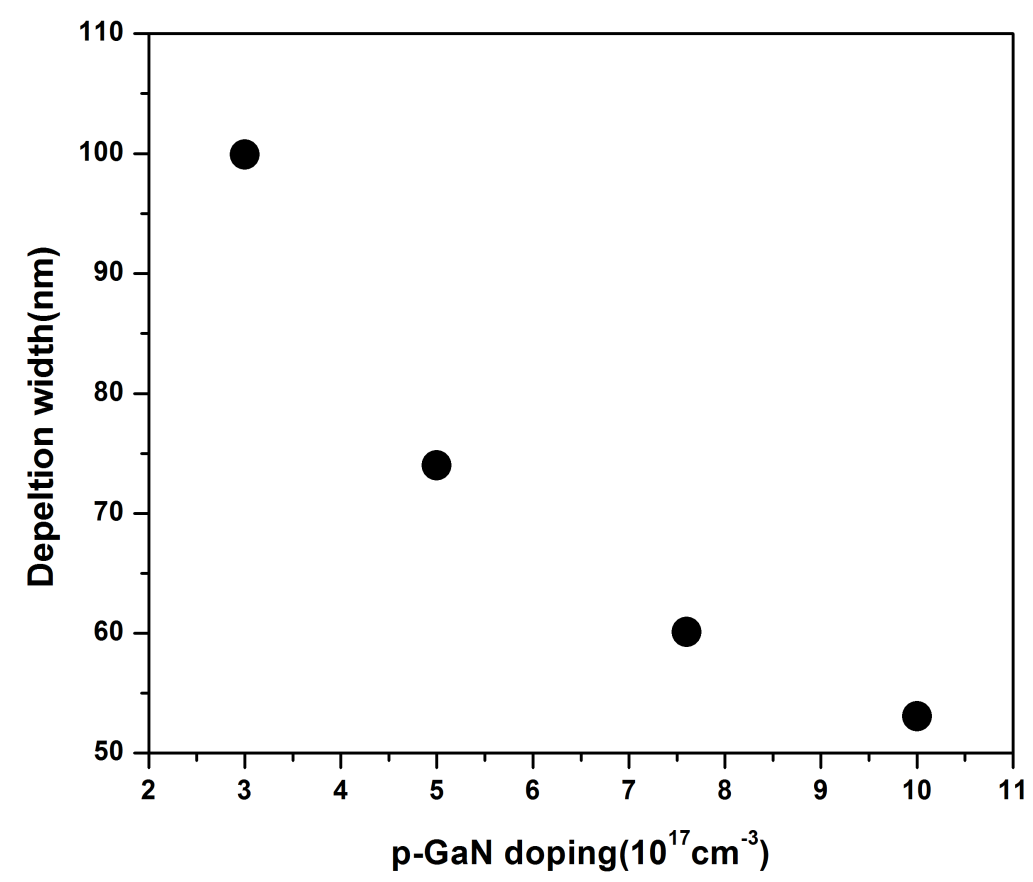

Figure 6.1: Calculated AZO/p-GaN junction depletion widths for varying hole concentration in the p-GaN layer. 
Given the challenges in incorporating p-type dopants into p-GaN, an alternative design approach has been studied. Earlier reports state that an ohmic could be achieved between AZO and p-GaN with the use of a nickel insertion layer. Having known that, Ni has a workfunction of around $4.1 \mathrm{eV}$ for 2 percent $\mathrm{Al}$ and band gap of around $3.43 \mathrm{eV}$. Simulation results indicate that a tunneling contact can be formed at Ni/AZO that varies with the thickness of Ni. IV and band diagrams resulting from a series of simulations at $\mathrm{Ni} / \mathrm{AZO}$ are as shown below:

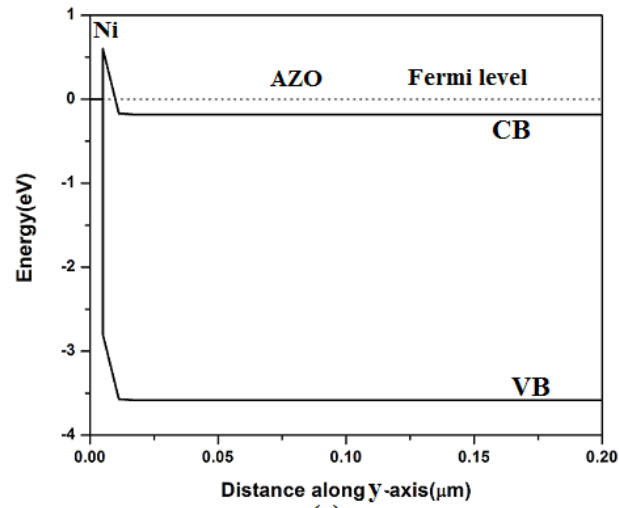

(a)

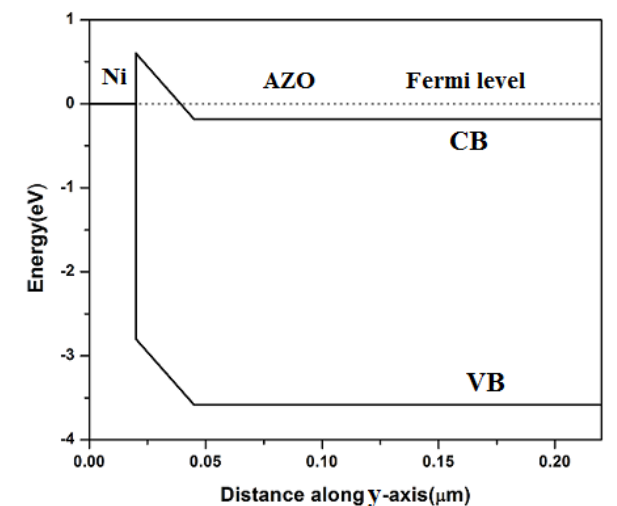

(b)

Figure 6.2: Band diagram indicating Ni/AZO junction.

Results indicate that, a tunneling contact can be achieved, and as the thickness of $\mathrm{Ni}$ on $\mathrm{AZO}$ varies from $2 \mathrm{~nm}$ to higher thickness the contact changes from ohmic to schottky contact. A tunneling barrier exists between $\mathrm{Ni}$ and AZO and the thickness of this barrier increases with increase in Ni thickness making the tunneling probability of carriers difficult.

\subsection{Analysis of $\mathrm{AZO}$ contact o p-GaN}

The AZO contact was then integrated via simulation on the LED structure. Initially the carrier concentration of AZO was take to be $5 \times 10^{20} \mathrm{~cm}^{-} 3$ and the thickness was taken to be 250nm. Such a structure had a current distribution as shown in fig 6.2.

Such a structure indicates current crowding at the mesa edge and could be explained by the fact that, 


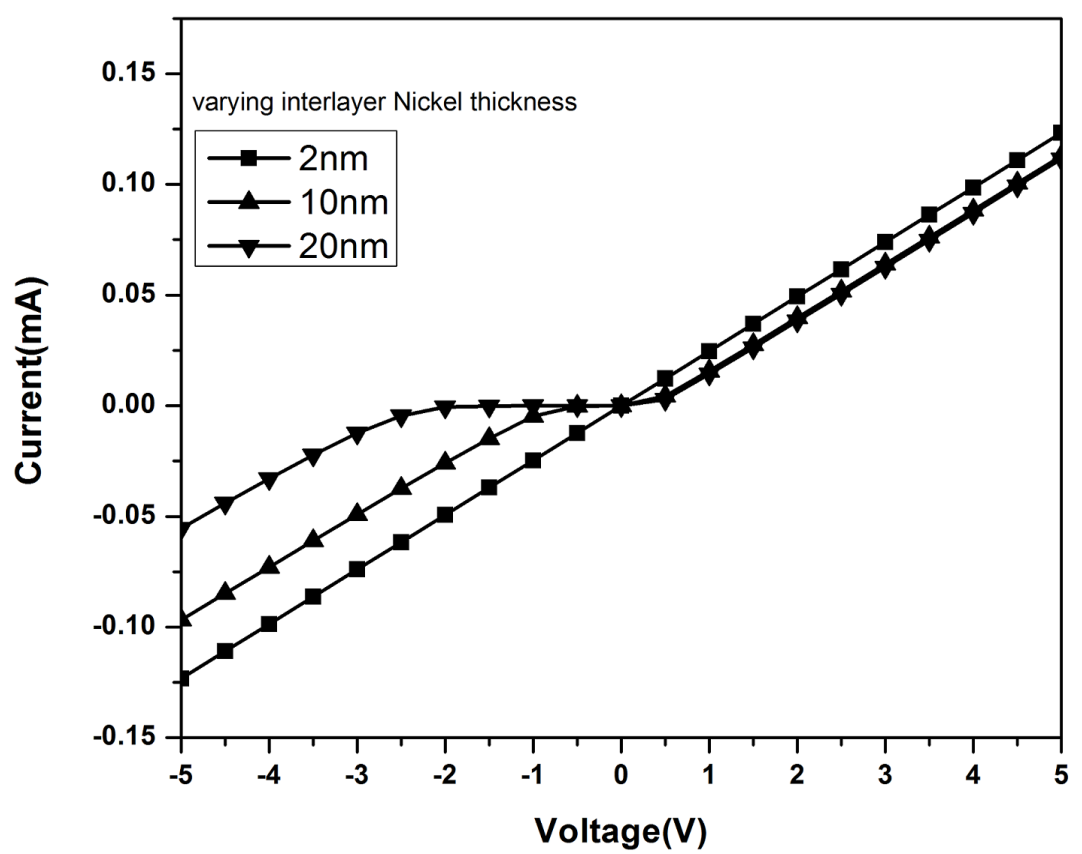

Figure 6.3: IV plots indicating Ni/AZO junction with varying Ni thickness.

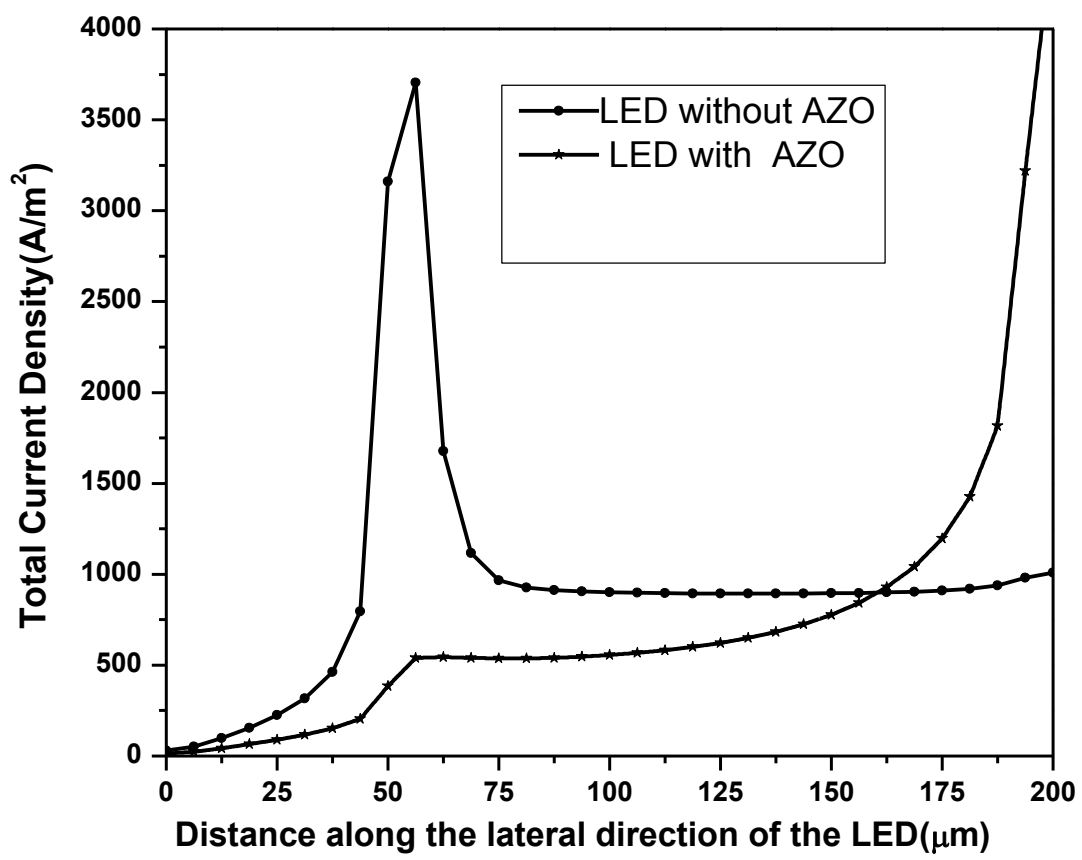

Figure 6.4: Current distribution in the LED mesa with AZO initial parameters. 


$$
\begin{aligned}
& \text { Resistivity of } A Z O=6.25 * 10^{-4} \Omega \mathrm{cm} \\
& \text { Resistivityofn-GaN }=3 * 10^{-2} \Omega \mathrm{cm} \\
& \frac{\rho_{t}}{t_{t}}=25 \Omega \\
& \frac{\rho_{n}}{t_{n}}=200 \Omega
\end{aligned}
$$

From equations 6.3 and 6.4 it is evident that the path along the TCO is least resistive and explains Figure 6.4 for current crowding at the mesa edge. From the analysis described in Chapter 5, to achieve a uniform current spreading it is desired that,

$$
\frac{\rho_{t}}{t_{t}}=\frac{\rho_{n}}{t_{n}}
$$

Now to have this condition satisfied, at any point of time if the conductivity of the material is fixed say in the order of $6.125 * 10^{-} 4 \Omega \mathrm{cm}$, the thickness of the AZO to have uniform current spreading should be equal to,

$$
t=\frac{\left(6.125 * 10^{-4}\right) * 1500 * 10^{-9}}{3.125 * 10^{-2}} m
$$

Thus for a given resistivity the thickness of AZO to have uniform current spreading is approximately 30nm. The current distribution of such a structure is as indicated in the figure 6.3 below.

The Figure:6.5 above depicts the current density distribution in the LED mesa with varying AZO thickness for a fixed resistivity. Thus for a given structure, if the conductivity of AZO is given, the thickness of the transparent conducting oxide needed to achieve an uniform current spreading can be calculated from the developed model. 


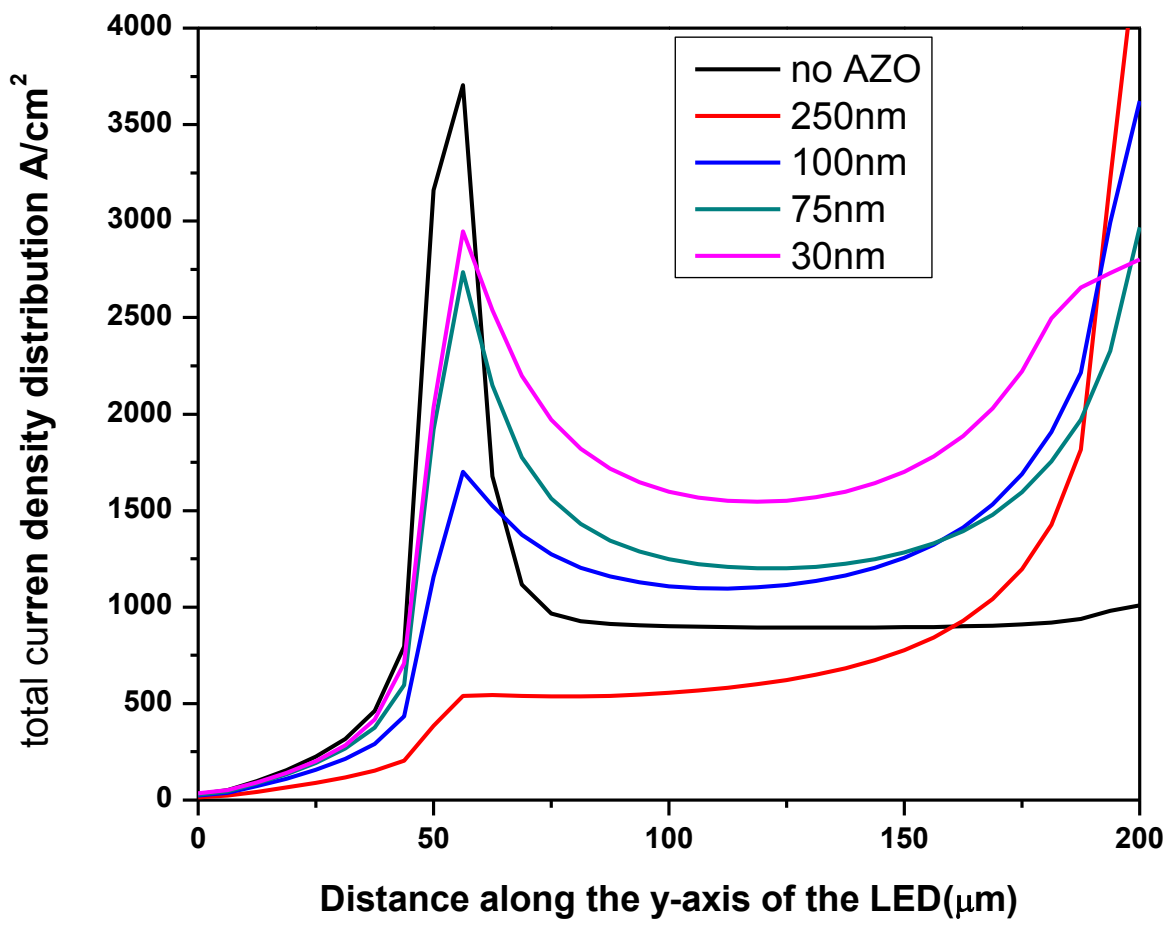

Figure 6.5: Current distribution in the LED mesa with varying thickness of AZO. 


\section{Chapter 7}

\section{Conclusions and Future work}

\subsection{Conclusions}

In this study, finite element analysis TCAD tool has been used to develop a model for GaN/InGaN based light emitting diodes. A TCAD model has been developed with the experimental parameters extracted from the MOVPE grown samples using Hall effect for carrier concenration, hall mobility and XRD from Indium and Aluminium concentration in InGaN, AlGaN, and the parameters like band gap, electron affinity, radiative recombination rate and effective masses taken from the literature.

The developed model has been validated by modeling different devices shown in the mask in Chapter 4, and comparing the simulated results with experimental results. The turn on voltage and series resistance have been extracted from the simulated IV's and the results were $+/-5 \%$ away from the experimental results.

On the developed model, the effectiveness of the electron blocking layer(EBL) has been studied by varying the thickness and composition and the results have been presented. The results indicate that for this this given LED structure $\mathrm{Al}_{0.1} \mathrm{Ga}_{0.9} \mathrm{~N}$ with a thickness of $10 \mathrm{~nm}$ had better confinement of electron-hole pairs to the active layers of the LED.

Given, that the electron affinity of $\mathrm{p}-\mathrm{GaN}$ is around $6.0 \mathrm{eV}$, achieving less resistive ohmic contacts has been a great challenge for researchers. Annealed Ni/Au contacts have been widely reported in the literature for use as ohmic contact to p-GaN. Upon, integration of $\mathrm{Ni} / \mathrm{Au}$ contact having known the fact that $\mathrm{n}-\mathrm{GaN}$ is more conductive than $\mathrm{p}-\mathrm{GaN}$, the 
current tries to shoot down at the edge of the p-contact and flows through the n-GaN to the n-contact. This current crowding results in heating effects and reduction in the extraction efficiency of the device due to the semitransparent nature associated with $\mathrm{Ni} / \mathrm{Au}$ contacts.

The GaN/InGaN LED has been analyzed using distributed resistance PSPICE modeling. An analytical model has been developed and indicates that depending on the resistance of p-GaN and n-GaN current crowding could occur either at the edge of the p-contact or p-mesa under the following conditions:

- If $\rho_{p} / \mathrm{t}_{p}>\rho_{n} / \mathrm{t}_{n}$, current crowds at the edge of the p-contact.

- If $\rho_{n} / t_{n}>\rho_{p} / t_{p}$, current crowds at the edge of the p-mesa.

- If $\rho_{p} / \mathrm{t}_{p}=\rho_{n} / \mathrm{t}_{n}$, a uniform current spreading in the mesa could be achieved.

Thus to have a uniform conducting a highly doped p-GaN with its thickness comparable to n-GaN has to be grown. Given, the challenges associated with growing such highly conductive p-GaN researchers have started exploring the use of transparent conducting contact to $\mathrm{p}$-GaN. Indium tin oxide (ITO) based contact to p-GaN has been widely reported in the literature. Having known the toxicity and the high cost involved in the growth of ITO films use of AZO as an alternated material to ITO has gained research interest[39][40].

AZO having an electron affinity of $4.1 \mathrm{eV}$ is expected to form a schottky contact with p-GaN [44] having a work function of $6.0 \mathrm{eV}$. In this study, an alternated method to achieve a tunneling contact between $\mathrm{AZO}$ and $\mathrm{p}-\mathrm{GaN}$ by the use of an intermediate nickel layer has been studied as a function of thickness of the nickel. Results, indicated that as the thickness of the nickel increases the contact changes from ohmic contact to schottky contact.

This Ni/AZO contact has been integrated on the LED structure and the analytical model developed has been used to achieve a uniform current spreading in the thickness. Thus, for a given conductivity of AZO, the thickness required could be determined using this model.

\subsection{Future Work}

Advancement to the model can be made by using sentaurus process that could take into account each of the individual steps involved in the fabrication of LEDs, including side wall 
angles and striations. The doping profile in this simulation has been considered to be a constant doping profile and the model can be modified to have a gaussian profile as is in the experimental materials. Development to the PSPICE based modeling should be done by taking into account the series resistance of the device. The model developed in this study had certain deviations from the experimental results. Possible reasons for this could be that, the polarization charges at the GaN/InGaN interface and the trap centers in the materials have not been activated due to lack of sufficient resources. Deep level transient spectroscopy (DLTS) could be used to obtain these charges and the non radiative recombination mechanism can be switched on to have a more closer match to the experimental results. Further development of the model could be made to integrate the optical modeling with the electrical modeling to have a more detailed understanding of the impact of various parameters on the internal and the external efficiencies. 


\section{References}

[1] A. Rizzi, "Private communications," 2001.

[2] Shuji Nakamura, Takashi Mukai, and Masayuki Senoh, "Candelaclass highbrightness ingan/algan double heterostructure blue light emitting diodes," Applied Physics Letters., vol. 64, pp. 1687, 1994.

[3] J. J. Wierer, D. A. Steigerwald, M. R. Krames, J. J. O'Shea, and M. J. Ludowise, "High-power algainn flip- chip light-emitting diodes," Applied Physics Letters., vol. 78, pp. 3379, 2001.

[4] Daniel L. Barton and Arthur J. Fischer, "Photonic crystals improve led efficiency," The International Society for Optical Engineering, 2006.

[5] N. Nakada, M. Nakaji, H. Ishikawa, T. Egawa, and M. Umeno, "Improved characteristics of ingan multiple-quantum-well light-emitting diode by gan/algan distributed bragg reflector grown on sapphire," Applied Physics Letters., vol. 76, pp. 1804, 2000.

[6] Jae-Hong Lim, Dae-Kue Hwang, Hyun-Sik Kim, Jin-Yong Oh, Jin-Ho Yang, R. Navamathavan, and Seong-Ju Parka, "Low-resistivity and transparent indium-oxide-doped zno ohmic contact to p-type gan," Applied Physics Letters., vol. 85, no. 25, 2004.

[7] Baaha E.A Saleh and Malvin Carl Teich, "Fundamentals of photonics," 1991.

[8] Gerhard Klimeck Alejandra J. Magana Saumitra Raj Mehrotra, Dragica Vasileska, "Carrier statistics lab learning materials," nano, 20010.

[9] E. Fred Schubert, Light Emitting Diodes, 2003.

[10] globalsino.com, "Semiconductors," .

[11] Naichia Yeh and Jen-Ping Chung, "High-brightness leds-energy efficient lighting sources and their potential in indoor plant cultivation," Renewable and Sustainable Energy Resources., vol. 13.

[12] Harry T. Whelan, James F. Connelly, Brian Hodgson, Lori Barbeau, A. Charles Post, George Bullard, Ellen V Buchmann, Mary Kane, Noel T. Whelan, Ann Warwick, and David Margolis, "Nasa light-emitting diodes for the prevention of oral mucositis in pediatric bone marrow transplant patients," Journal of Clinical laser Medicine and Surgery., vol. 20, no. 6, 2002. 
[13] Nick Holonyak and S. F. Bevacqua, "Coherent (visble) light emission from ga(as1-xpx) junctions," Applied Physics Letters., vol. 1, no. 4, pp. 82-83, 1962.

[14] H.P. Maruska and J.I. Pankove, "Efficiency of $\operatorname{gaas}_{1-x} \mathrm{p}_{x}$ electroluminescent diodes," Solid State Electronics, vol. 10, no. 9, 1967.

[15] I. Akasaki, H. Amano, M. Kito, and K. Hiramatsu, "Photoluminescence of mg-doped ptype gan and electroluminescence of gan p-n junction led," Journal of Lumminescence., vol. 48, pp. 666-670, 1991.

[16] H.P. Maruska and J.J. Tietjen, "The preparation and properties of vapor deposited single crystalline gan," Applied Physics Letters., vol. 15, no. 10, 1969.

[17] Miller Ea Pankove Ji and BERKEYHE.JE, "Gan electroluminscent leds," RCA review, vol. 32, no. 3, pp. 383, 1971.

[18] Rhines W. C. Maruska H. P. and Stevenson D. A, "Preparation of mg-doped gan diodes exhibiting violet electroluminescence," Materials Research Bulletin, vol. 7, no. 8, pp. 777-781, 1972.

[19] K Hiramatsu H. Amano, M. Kito and I. Akasaki, "P-type conduction in mg-doped gan treated with low-energy electron beam irradiation," vol. 28, pp. L2112-L2114, 1989.

[20] Nakamura S, Senoh M, Iwasa N, and Nagahama S, "High-brightness ingan blue, green and yellow light-emitting diodes with quantum well structures," Journal of Applied Physics., vol. 34, pp. 797-799, 1995.

[21] June O Song, Jun-Seok Ha, , and Tae-Yeon Seong, "Ohmic-contact technology for ganbased light-emitting diodes: Role of p-type contact," IEEE transactions on Electronic Devices., vol. 57, no. 1, 2010.

[22] M. Koike, S. Yamasaki, S. Nagai, N. Koide, S. Asami, H. Amano, , and I. Akasaki, "High-quality gainn/gan multiple quantum wells," Applied Physics Letters., vol. 68, pp. 1403, 1996.

[23] M. Boroditsky, T. F. Krauss, R. Coccioli, R. Vrijen, R. Bhat, and E. Yablonovitch, "Light extraction from optically pumped light - emitting diode by thin - slab photonic crystals," Applied Physics Letters., vol. 75, pp. 8, 1999.

[24] S. Kobayashia S. Yamasakib S. Nagaib J. Umezakib M. Koikeb Masanori Murakam Yasuo Koide, H. Ishikawaa, "Dependence of electrical properties on work functions of metals contacting to p-type gan," Applied Surface Science, vol. 117, no. 2, pp. 373-379, 1997.

[25] D.W. Kim, Y.J. Sung, J.W. Park, and G.Y. Yeom, "A study of transparent indium tin oxide (ito) contact to p-gan," Thin Solid Films., pp. 398-399, 2001.

[26] Mansun Chan Richard S. Muller, Theodore I. Kammins, "Device electronics for integrated circuits," 2002. 
[27] Adrian Kitai, "Principles of solar cells, leds and diodes: The role of the p-n junction," 2002.

[28] Pallab Bhattacharya, "Semiconductor optoelectronic devices," 1996.

[29] Joachim Piprek, "Semiconductor optoelectronic devices:introduction to physics and simulation," 2003.

[30] Synopsys, "Sentaurus," 2010.

[31] V.M Bermudez, "Study of oxygen chemisorption on the gan(0001)-(11) surface," Journal of Applied Physics., vol. 80, no. 2, pp. 1190, 1996.

[32] C.I. Wu and A. Kahn, "Investigation of the chemistry and electronic properties of metal/gallium nitride interfaces," Journal of Vaccum Science and Technology, vol. 16, no. 4, pp. 2218, 1998.

[33] J.I Pankove and H. Schade, "Photoemission from gan," Applied Physics Letters., vol. 25, no. 1, pp. 53, 1974.

[34] G. C. Chi P. L. Koh M. J. Jou C. M. Chang C. C. Liu J. K. Sheu, Y. K. Su and W. C. Hung, "Photoemission from gan," Applied Physics Letters., vol. 74, no. 16, pp. 2340-2342, 1999.

[35] M. O. Jones D. V. MorGaNb P. P. Edwards, A. Porch and R. M. Perks, "Basic materials physics of transparent conducting oxides," Dalton Transactions, , no. 19, pp. 2995-3002, 2004.

[36] Y. K. Su T. K. Tsai C. S. Chang S. C. Shei C. W. Kuo Y. C. Lin, S. J. Chang and S. C. Chen, "Ingan/gan light emitting diodes with ni/au, ni/ito and ito p-type contacts," Solid State Electronics, vol. 47, pp. 849-853, 2003.

[37] Ho Won Jang Soo Young Kim and Jong-Lam Lee, "Effect of an indium-tin-oxide overlayer on transparent ni/au ohmic contact on p-type gan," Applied Physics Letters., vol. 82, no. 61, 2003.

[38] Jiunn-Yi Chu Kow-Ming Chang and Chao-Chen Cheng, "Highly reliable gan-based light-emitting diodes formed by p-in0:1ga0:9n-ito structure," IEEE Photonics Technology Letters, vol. 16, no. 8, 2004.

[39] Gordon R G, "Criteria for choosing transparent conductors," Materials Research Bulletin, vol. 25, pp. 52, 2000.

[40] V. E. Sandanaa D. J. Rogers, F. Hosseini Teherani and M. Razeghi, "Zno thin films \& nanostructures for emerging optoelectronic applications," The International Society for Optical Engineering, vol. 7605, 2010. 
[41] Lee Soon Park Sang Hern Lee Jong Hyeob Baek Tae Hoon Kim, Young-Gu Ju and Young Moon $\mathrm{Yu}$, "Enhanced optical output power of tunnel junction gan-based light emitting diodes with transparent conducting al and ga-codoped zno thin films," Journal of Applied Physics., vol. 49, 2010.

[42] M. L. Lee C. C. Hu C. K. Hsieh C. J. Tun, J. K. Sheu and G. C. Chi, "Effects of thermal annealing on al-doped zno films deposited on p-type gallium nitride," Journal of Electrochemical Society, vol. 153, no. 4, 2006.

[43] M. K. Fung X. Jiang, F. L. Wong and S. T. Lee, "Aluminum-doped zinc oxide films as transparent conductive electrode for organic light-emitting devices," Applied Physics Letters., vol. 83, no. 1875, 2003.

[44] Sang Hern Lee and Young Moon Yu, "Characterization of transparent conducting aldoped zno thin films and device applications," Journal of Korean Physics Society, vol. 51, pp. S79-S83, 2007. 